\title{
THE JCMT GOULD BELT SURVEY: DENSE CORE CLUSTERS IN ORION A
}

\author{
J. Lane ${ }^{1,2}$, H. KirK ${ }^{1}$, D. Johnstone ${ }^{1,2,3}$, S. Mairs ${ }^{1,2}$, J. Di Francesco ${ }^{1,2}$, S. Sadavoy ${ }^{4}$, J. Hatchell ${ }^{5}$, D. S. Berry ${ }^{6}$, \\ T. JENNESS ${ }^{3,7}$, M. R. HOGERHEIJDE ${ }^{8}$, AND D. WARD-THOMPSON ${ }^{9}$ \\ The JCMT Gould Belt Survey Team ${ }^{10}$ \\ ${ }^{1}$ NRC Herzberg Astronomy and Astrophysics, 5071 West Saanich Road, Victoria, BC, V9E 2E7, Canada \\ 2 Department of Physics and Astronomy, University of Victoria, Victoria, BC, V8P 1A1, Canada \\ ${ }^{3}$ Joint Astronomy Centre, 660 N. A'ohōkū Place, University Park, Hilo, Hawaii 96720, USA \\ ${ }_{5}^{4}$ Max Planck Institute for Astronomy, Königstuhl 17, D-69117 Heidelberg, Germany \\ ${ }^{5}$ Physics and Astronomy, University of Exeter, Stocker Road, Exeter EX4 4QL, UK \\ ${ }^{6}$ East Asian Observatory, 660 N. A'ohōkū Place, University Park, Hilo, Hawaii 96720, USA \\ ${ }^{7}$ LSST Project Office, 933 N. Cherry Avenue, Tucson, AZ 85719, USA \\ ${ }^{8}$ Leiden Observatory, Leiden University, P.O. Box 9513, 2300 RA Leiden, The Netherlands \\ 9 Jeremiah Horrocks Institute, University of Central Lancashire, Preston, Lancashire, PR1 2HE, UK \\ Received 2016 April 29; revised 2016 August 30; accepted 2016 September 1; published 2016 December 6
}

\begin{abstract}
The Orion A molecular cloud is one of the most well-studied nearby star-forming regions, and includes regions of both highly clustered and more dispersed star formation across its full extent. Here, we analyze dense, star-forming cores identified in the 850 and $450 \mu \mathrm{m}$ SCUBA-2 maps from the JCMT Gould Belt Legacy Survey. We identify dense cores in a uniform manner across the Orion A cloud and analyze their clustering properties. Using two independent lines of analysis, we find evidence that clusters of dense cores tend to be mass segregated, suggesting that stellar clusters may have some amount of primordial mass segregation already imprinted in them at an early stage. We also demonstrate that the dense core clusters have a tendency to be elongated, perhaps indicating a formation mechanism linked to the filamentary structure within molecular clouds.
\end{abstract}

Key words: Interstellar medium (ISM), Nebulae - stars: formation - submillimeter: ISM

\section{INTRODUCTION}

While the Shu et al. (1987) model provides a framework to understand many aspects of the formation of a single, lowmass, isolated star, much remains to be done to expand a similar understanding to a wider range of systems. Most lowmass stars and nearly all high-mass stars form in clustered environments (Lada \& Lada 2003; Zinnecker \& Yorke 2007), making the understanding of the clustered mode of star formation important. Nevertheless, there is little consensus on the formation process of either high-mass stars or stellar clusters. One avenue that will help to guide models of cluster formation is a careful characterization of the basic properties of clustered systems at early times, which will put constraints on the initial conditions of such systems. Our goal in this paper is to present one such characterization of dense core clusters in Orion A, which can easily be reproduced in other star-forming environments, allowing for a quantification of the range of typical cluster properties held by an ensemble of clouds.

There have been many studies of the clustering properties of young stars, including Gutermuth et al. (2009, hereafter G09) and Kirk \& Myers (2011, hereafter KM11), both of which analyzed samples of nearby star-forming regions (mostly within $1000 \mathrm{pc}$ for the former and all within $500 \mathrm{pc}$ for the latter), thereby ensuring that even low-mass stars were

\footnotetext{
${ }^{10}$ The full members of the JCMT Gould Belt Survey Consortium are P. Bastien, D. S. Berry, D. Bresnahan, H. Broekhoven-Fiene, J. Buckle, H. Butner, M. Chen, A. Chrysostomou, S. Coude, M. J. Currie, C. J. Davis, E. Drabek-Maunder, A. Duarte-Cabral, J. Di Francesco, M. Fich, J. Fiege, P. Friberg, R. Friesen, G.A. Fuller, S. Graves, J. Greaves, J. Gregson, J. Hatchell, M.R. Hogerheijde, W. Holland, T. Jenness, D. Johnstone, G. Joncas, J.M. Kirk, H. Kirk, L.B.G. Knee, S. Mairs, K. Marsh, B.C. Matthews, G. MoriartySchieven, J.C. Mottram, C. Mowat, K. Pattle, J. Rawlings, J. Richer, D. Robertson, E. Rosolowsky, D. Rumble, S. Sadavoy, N. Tothill, H. Thomas, S. Viti, D. Ward-Thompson, G.J. White, J. Wouterloot, J. Yates, and M. Zhu.
}

included. One intriguing result from KM11 was that young (1-2 Myr), small (20-40 member), and sparse (less than $100 \mathrm{stars}^{-2}$ ) groupings of stars showed evidence of mass segregation through the presence of a centrally located, most massive cluster member. It would be difficult for such an arrangement to be fully caused by dynamical interactions after the stars had formed, given the young ages of the systems. A later analysis of numerical simulations of the formation of small stellar groups also showed that mass segregation is present as early in the simulation as it was measurable (Kirk et al. 2014). These results raise the question of how the mass in a cluster-forming region is arranged before star formation begins. Mass segregation, particularly in clusters of dense cores, is therefore an important factor in understanding the initial conditions of star formation.

We study dense cores (structures containing roughly $1 M_{\odot}$ of material within $\sim 0.1 \mathrm{pc}$, as in Bergin \& Tafalla 2007) in the Orion A molecular cloud. Orion A is a $\sim 10^{5} M_{\odot}$, nearby ( 415 pc, Menten et al. 2007; Kim et al. 2008) cloud that is forming both high- and low-mass stars (as reviewed in Bally 2008, p. 459). The brightest submillimeter feature in Orion A is the integral shaped filament (ISF), in the northern part of the cloud (e.g., Bally et al. 1987; Johnstone \& Bally 1999). The ISF contains the Orion Nebula Cluster $(\mathrm{ONC})$, a large cluster of young stars including several O stars (Hillenbrand 1997). The Orion A cloud also contains many sites of more dispersed star formation, extending approximately $30 \mathrm{pc}\left(\sim 4^{\circ}\right)$ to the southeast from the center of the ISF, in an area known as Orion A South (Bally 2008, p. 459). This southern portion of Orion A, also known as L1641, was recently studied by Polychroni et al. (2013) using Herschel observations, showing that the dense cores, especially the more massive dense cores, have a strong tendency to be associated 
with filamentary structures. With its large number of dense cores and varied clustering envioronments, Orion A provides the ideal laboratory to explore mass segregation in dense core clusters. To perform this analysis, we use SCUBA-2 (Holland et al. 2013) observations at 450 and $850 \mu \mathrm{m}$ taken as part of the JCMT Gould Belt Survey (Ward-Thompson et al. 2007). An analysis of dense cores and filamentary structure found in Orion A North were published by Salji et al. (2015a) and Salji et al. (2015b), respectively, while an analysis of the fragmentation stability of dense gas in southern Orion A is given by Mairs et al. (2016). The clustering analysis presented here uses a similar methodology to a recent clustering analysis of Orion B by our group (Kirk et al. 2016b, hereafter K16), and we later discuss the implications of finding similar results in both Orion clouds.

\section{OBSERVATIONS}

Salji et al. (2015a) and Mairs et al. (2016) present a "first look" at the SCUBA-2 data across the northern and southern portions of Orion A, respectively, and the data reduction methods used in each of their analyses are given in their papers. In short, the northern Orion A analysis by Salji et al. (2015a) used only the first portion of data available in the region (i.e., a smaller area and shallower sensitivity than the final data set). These data were reduced using an earlier reduction method, Internal Release 1 (IR1), which had larger pixel sizes (6" versus the current $3^{\prime \prime}$ ) and did not recover extended emission as well as the current reduction method. The southern portion of Orion A analyzed by Mairs et al. (2016) includes the full GBS data obtained in this region and applies the present best GBS reduction method, Legacy Release 1 (LR1), for the data reduction. Our present analysis of the entire Orion A complex also uses the full GBS data set, but only uses a part of the full LR1 data reduction procedure, due to availability at the time of our analysis. We outline the differences between our reduction method and LR1 below, and highlight the resultant differences in the final maps.

Individual observations at 850 and $450 \mu \mathrm{m}$ were made using fully sampled $30^{\prime}$ diameter circular regions (PONG 1800 mapping mode, Bintley et al. 2014); larger regions were mosaicked with overlapping scans. The data were reduced as part of the GBS LR1 using an iterative map-making technique (makemap in SMURF, Chapin et al. 2013), and gridded to $3^{\prime \prime}$ pixels at $850 \mu \mathrm{m}$ and $2^{\prime \prime}$ pixels at $450 \mu \mathrm{m}$. The iterations were halted when the map pixels, on average, changed by $<0.1 \%$ of the estimated map rms. This reduction is referred to as the automask reduction. The final GBS LR1 data products are created through a second round of reductions, wherein a signalto-noise ratio mask is produced based on a mosaic of the automask-reduced data, to define areas of probable emission (see Mairs et al. 2016 for more details or Kirk et al. 2016a for a description of the same data reduction process in Orion B). This second reduced map is referred to as the external mask reduction. Both the automask and external mask reductions include a spatial filter of $600^{\prime \prime}$, limiting robust flux recovery to sources with a Gaussian FWHM less than 2!5. sources between $2 ! 5$ and 7.5 will be detected, but both the flux density and the size are underestimated because Fourier components with scales greater than $5^{\prime}$ are removed by the filtering process. Use of an external mask allows for somewhat better source recovery than the initial automask reduction, as demonstrated by Mairs et al. (2015). Testing with artificial sources in Mairs et al.
(2015) shows that compact and brighter sources are reasonably well-recovered even with an automask-only reduction, whereas fainter and more diffuse sources tend to have underestimated sizes and flux densities. Comparison of SCUBA-2 and Herschel $500 \mu \mathrm{m}$ data in the L1495 region of Taurus also shows that SCUBA-2 is most sensitive to the coldest and densest gas structures (Ward-Thompson et al. 2016).

We use only the automask-reduced mosaics of Orion A here. This choice was motivated by the data available at the time of analysis. While the automask mosaic is insufficient for a detailed study of core mass functions, since faint sources would have underestimated fluxes, this reduction method is sufficient for clustering analyses. Our requirements for this analysis are to be able to identify dense cores, and, within sub-regions of the map, rank the cores by flux (in particular, to identify the highest flux density dense core). Accuracy on the absolute flux density values (or any size estimate) is not important for our analysis. Furthermore, the small suppression of larger scale, faint, and diffuse emission in complex regions such as the integral shaped filament, likely provides a slight advantage in simplifying the separation of this complex emission into dense cores. Figure 1 shows the $850 \mu \mathrm{m}$ map that we analyzed, highlighting the variety in emission structure along the cloud.

We include a copy of the automask mosaic used in our analysis at https://doi.org/10.11570/16.0008.

\section{SOURCE EXTRACTION}

We applied two different source identification methods across our Orion A map to create independent uniform catalogs for our analysis. These two methods were the getsources (Men'shchikov et al. 2012) and FellWalker(Berry 2015) algorithms. In the getsources algorithm, ${ }^{11}$ cores are extracted by convolving an image on multiple spatial scales to find cores and filter out smallscale noise and large-scale structure. FellWalker identifies cores by following flux density gradients toward peaks to define the boundaries of cores. Getsources is a multi-wavelength extraction method and can therefore make use of both 850 and $450 \mu \mathrm{m}$ data simultaneously, while FellWalker was run only on the $850 \mu$ m data. After removing sources that were likely noise artifacts, the final getsources catalog contained 919 cores and the final FellWalker catalog contained 773 cores. The details of getsources, FellWalker, and the automated procedure to eliminate spurious sources in each are discussed in more detail in Appendices A.1.1 and A.1.2 respectively. We found that getsources did a better job of separating small, compact cores from the underlying large-scale cloud structure and we therefore use this catalog for the analysis presented in this paper. In Appendices A.1.3, A.2, and A.3, we present comparable analyses using the FellWalker-based catalog.

Creating our own dense core catalog across Orion A was necessary to ensure that cores were identified uniformly across the entire cloud. The differing sensitivities of the maps and also core identification algorithms used by Salji et al. (2015a) and Mairs et al. (2016) in the north and south could result in biases in our analysis if we attempted to combine their two core catalogs. Additionally, we note that the analysis of Mairs et al. (2016) was not focussed on identifying discrete dense cores, but instead on characterizing the fragmentation of larger scale features in the cloud, and hence their catalog would not be

11 We used version 1.140127. 


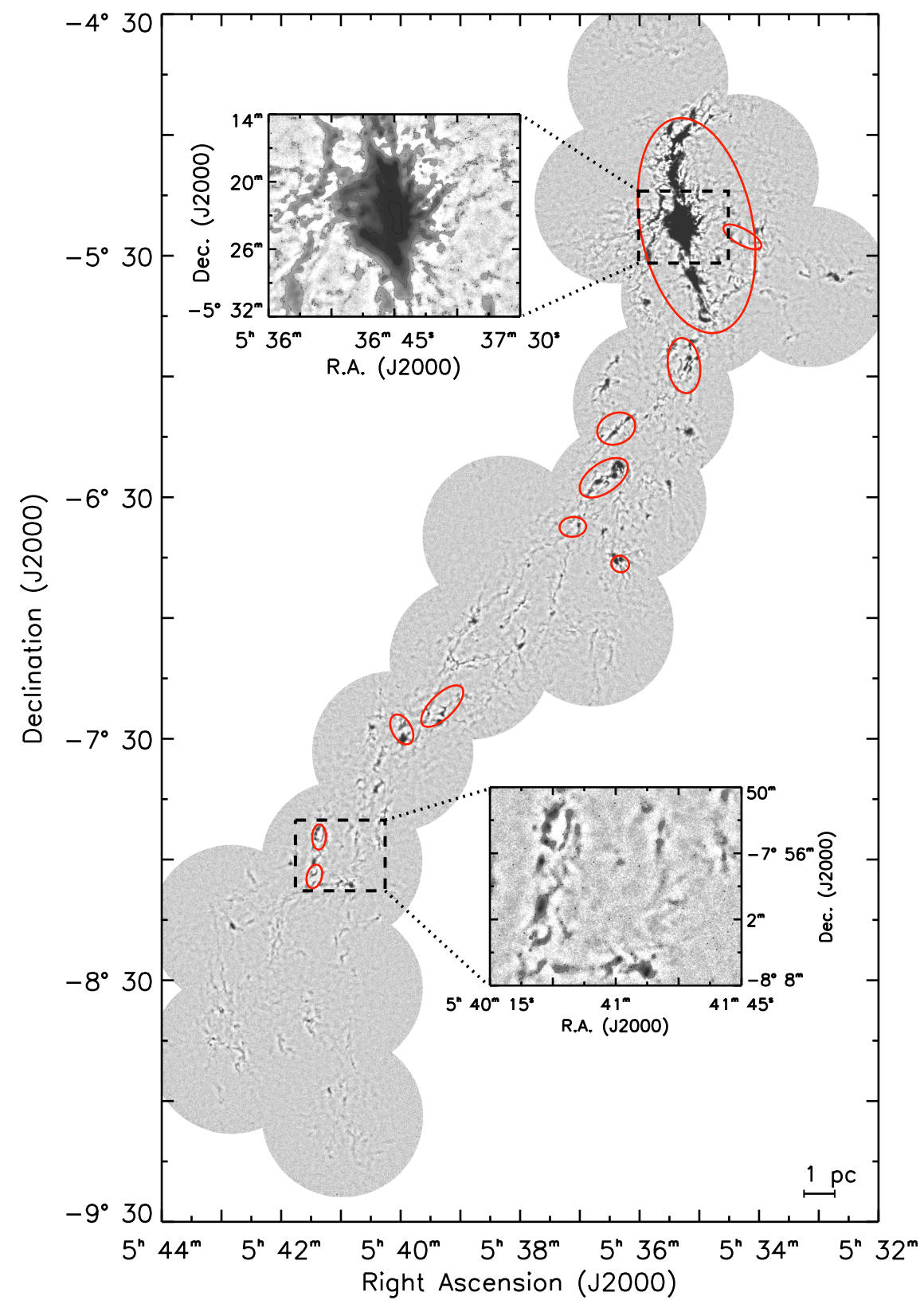

Figure 1. $850 \mu \mathrm{m}$ flux density data for Orion A, shown with logarithmic scaling. The contour lines are placed at $0.01,0.1,0.5$, and $2.5 \mathrm{Jy}$ arcsec ${ }^{-2}$. The red circles show the approximate locations of the MST-based clusters identified in Section 4. The two insets show selected $22^{\prime} .5$ by $18^{\prime}$ regions. The inset near the top of the figure shows the bright ISF in the northern part of the cloud. This structure is contrasted by the sparser structure in the south, shown in the bottom inset.

directly applicable to our science goals. Source identification is particularly difficult around the ISF in the northern part of Orion A, where many compact peaks of emission lie clustered within a larger zone of extended emission. By running the two independent core identification algorithms listed above, we can additionally test the robustness of our analysis. Despite large differences in the overall catalogs (the total number of cores and core boundaries, especially in the ISF), Appendix A demonstrates that we find similar results for our clustering analysis using either catalog.

\subsection{Protostars}

After creating our getsources-based dense core catalog, we classified each core as being starless or protostellar based on the Spitzer catalog of Megeath et al. (2012) and the Herschel catalog of Stutz et al. (2013). From the Megeath et al. (2012) catalog, we used only the protostars deemed "reliable," i.e., sources that were too faint at $24 \mu \mathrm{m}$ or went undetected in shorter wavebands were not included. We defined a dense core as protostellar if one or more Spitzer or Herschel protostars were found within one beam radius $\left(77^{\prime \prime} 25\right)$ of the central core peak. The Spitzer catalog was sufficient to identify most of the protostellar cores. Indeed, only five cores were deemed protostellar based solely on the Herschel catalog. Our protostar-core association criterion is more stringent than the one applied in K16 because Orion A has a much higher surface density of protostars. With our criterion, 814 of the cores are deemed starless, while 105 are classified as protostellar, i.e., roughly $11 \%$ of the cores are protostellar. For comparison, Mairs et al. (2016) found 75 of 359 islands and 103 of 431 fragments contained at least one protostar (i.e., $21 \%$ to $24 \%$ 
protostellar) in the southern portion of Orion A, while Salji et al. (2015a) found 48 protostellar cores and 432 starless and prestellar cores around the ISF (i.e., 10\% protostellar). We note that the Mairs et al. (2016) protostellar fraction is significantly higher than the other two cases because any structure that had a protostar located somewhere within its boundary was classified as protostellar, rather than restricting the protostellar classification to those objects that have a protostar near the brightest peak within the object. We also note that additional candidate YSOs based on recent radio (Forbrich et al. 2016) or nearinfrared (Meingast et al. 2016) detections were not included in our analysis, nor in that of Salji et al. (2015a) or Mairs et al. (2016), implying that all of the protostellar fractions listed here are lower limits.

\subsection{Core Masses}

Using the total flux density measured for each core at $850 \mu \mathrm{m}$, we can also estimate the core masses. Assuming a constant dust temperature and opacity, the conversion between flux density and mass is given by

$$
\begin{aligned}
M= & 1.30\left(\frac{S_{850}}{1 \mathrm{Jy}}\right)\left(\frac{\kappa_{850}}{0.012 \mathrm{~cm}^{2} \mathrm{~g}^{-1}}\right) \\
& \times\left(\exp \left(\frac{17 \mathrm{~K}}{T_{d}}\right)-1\right)\left(\frac{D}{450 \mathrm{pc}}\right)^{2} M_{\odot},
\end{aligned}
$$

where $S_{850}$ is the total flux density at $850 \mu \mathrm{m}, \kappa_{850}$ is the dust opacity at $850 \mu \mathrm{m}, T_{d}$ is the dust temperature, and $D$ is the distance. For consistency with Salji et al. (2015a) and Mairs et al. (2016), we assume a constant dust grain opacity of $0.012 \mathrm{~cm} \mathrm{~g}^{-1}$, and a distance to Orion A of $450 \mathrm{pc}$ (e.g., Muench et al. 2008). Also following Mairs et al. (2016), we assume a dust temperature of $15 \mathrm{~K}$. We note that the assumption of a constant temperature is necessarily approximate. For example, Salji et al. (2015a) and Johnstone \& Bally (2006) find evidence of variations in temperature across the northern and southern portions of Orion A respectively, and a comparison of the $450-850 \mu \mathrm{m}$ flux density ratio by D. Rumble et al. (2016, in preparation) suggests that temperatures are generally higher in the north, as is also seen in the Herschel-based analysis of Lombardi et al. (2014). The cores most poorly represented by a fixed temperature are likely to be some of the protostellar cores, where a higher temperature would be more appropriate. With the assumption of a constant $15 \mathrm{~K}$ temperature, the median core mass is $0.8 M_{\odot}$ in both the getsources-based and FellWalker-based catalogs, with cores as small as $0.06 M_{\odot}$ identified. In our analysis below, where the flux- or mass-ranking of cores is important to the results, we examine both the entire core population and also the starless core subsample separately. In Appendix C, we re-run all of the analyses presented in the main paper, using Herschel-based temperatures from Lombardi et al. (2014) to estimate the massranking of the cores. In all cases, we reach similar conclusions as are presented in the main text. As we outline in Appendix C, the Lombardi et al. (2014) temperatures were estimated without the inclusion of long wavelength data, which could lead us to assign artifically high temperature estimates of some dense cores, since Herschel is more sensitive to diffuse warm dust than SCUBA-2. Since diffuse warm dust might not be distributed uniformly across Orion A, our main analysis uses the aforementioned flux-ranking of the dense cores, while mass-ranking based on Herschel temperatures is left to Appendix $\mathrm{C}$ to provide an additional test to confirm our results.

\section{CLUSTERING ANALYSIS}

As seen in Figure 1, the dense cores in Orion A inhabit a variety of environments, ranging from highly clustered in the north around the ISF to more isolated in the south. High-mass star formation is ongoing in the north, where the density of gas and dust is the highest, while in the south there is more distributed low-mass star formation (Bally 2008, p. 459). Despite these differences in star-forming activity, there are visible clusters of dense cores in both the north and south of the cloud, albeit on different spatial scales. Clusters in the south are often isolated and more dispersed/extended than clusters in the north. Bressert et al. (2010) showed that protostellar clustering, not only in Orion but across the whole Gould Belt, exists along a spectrum of spatial densities, suggesting the distinction between clustered and isolated star formation is necessarily relative, rather than absolute. A major difficulty in identifying clusters in a cloud as large and varied as Orion $\mathrm{A}$ is determining a consistent method that works well for all clustering scales that are present. For this reason, we use a minimal spanning tree (MST) method following G09 and KM11 (see also Cartwright \& Whitworth 2004, for a completely different application of the MST in analyzing clustered structures). The advantage of the MST method is that it is able to pick out the relative overdensities that represent clusters. The other feature of the MST which is especially advantageous for our analysis is that there is no preference imposed on identifying round clusters: since star formation is thought to be strongly associated with filaments (e.g., André et al. 2014, p. 27), dense core clusters may also show some elongation.

\subsection{Using an $M S T$}

An MST is formed by connecting each core to its nearest neighbor and ensuring that all cores are connected together continuously, as shown in the left panel of Figure 2. The connections between cores are known as branches, and the total length of all branches in an MST is minimized such that all cores are connected together as efficiently as possible. In an MST diagram, clusters are groups of cores connected by shorter branch lengths, a characteristic that can be used to extract clusters. G09 created a criterion for cluster membership based on the distribution of MST branch lengths. Figure 3 shows the cumulative distribution of MST branch lengths for the southern portion of Orion A (see the following paragraph). The curve has two distinct parts: a steep, initial increase, followed by a turnover to a shallow gradient. This is the characteristic shape of the branch length distribution for cluster-forming regions, as shown in G09. The steep part of the distribution at shorter branch lengths represents clustered environments, while the longer branch lengths at the flat end of the distribution represent isolated cores as well as the clustercluster connections. G09 defines $L_{\text {crit }}$ as the intersection between linear fits to both ends of the distribution. Removing all branches longer than $L_{\text {crit }}$ leaves the spatially dense regions connected together, which represent clusters, as seen in the 

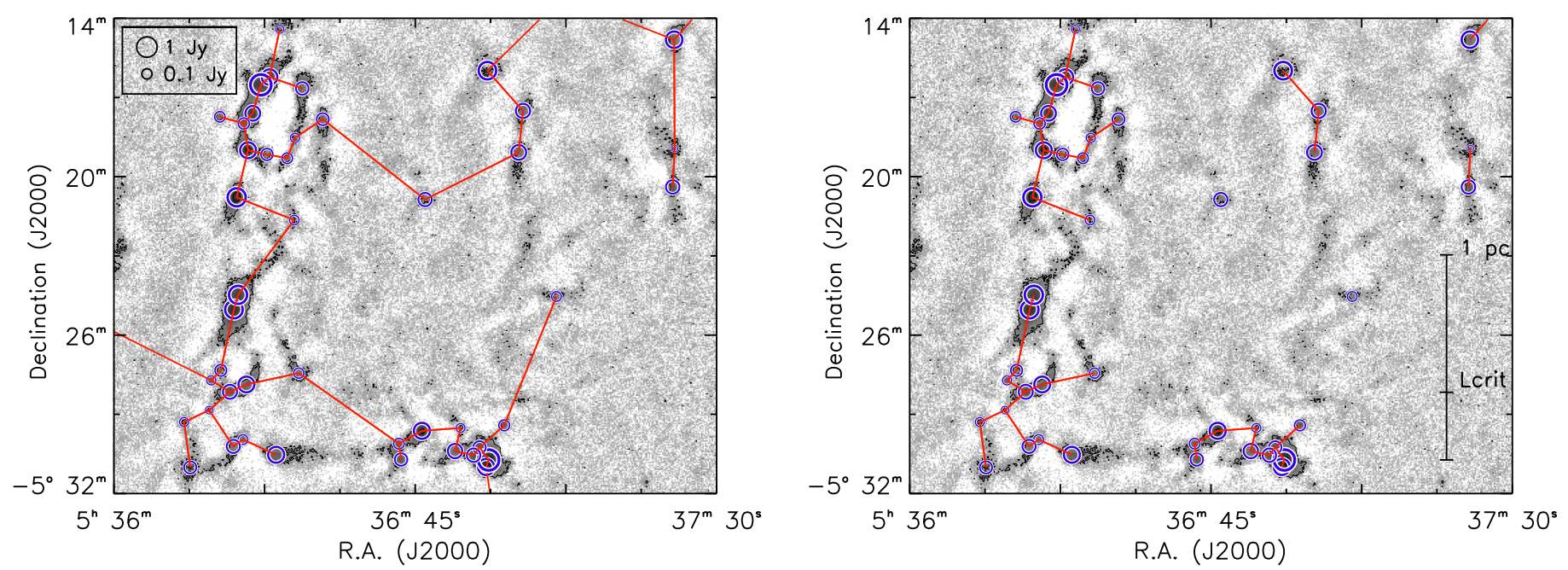

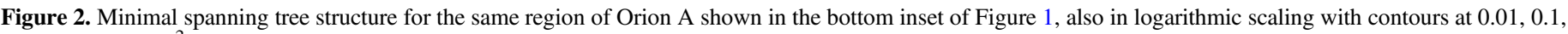

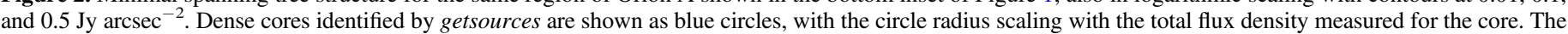

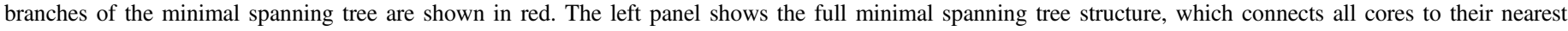

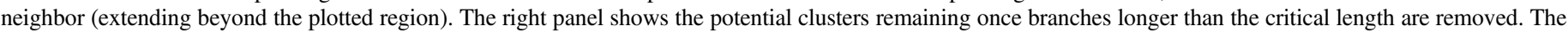
long scale bar indicates a length of $1 \mathrm{pc}$ assuming a distance of $450 \mathrm{pc}$, while the shorter segment shows the value of $L_{\text {crit }}$ determined, i.e., 0.36 pc.

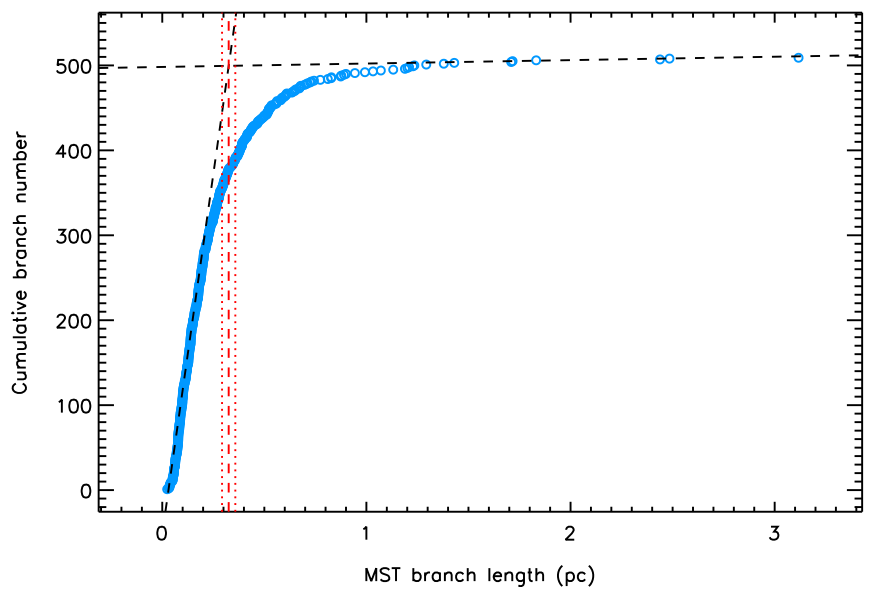

Figure 3. MST branch lengths measured in Orion A, with ISF cores removed. The cumulative number of branches with a given length or smaller are indicated by the blue circles. The dashed black lines show the best linear fits to the two ends of the branch length distribution, while the thick dashed red line shows the best-fit $L_{\text {crit }}$ value (i.e., the branch length at which the two blue lines intersect). The dotted red vertical lines show the range in $L_{\text {crit }}$ values resulting from acceptable linear fits to the distribution.

right panel of Figure 2. We estimate the uncertainty in $L_{\text {crit }}$ by finding the steepest and shallowest possible linear fits to each of the cumulative distributions and re-calculating $L_{\text {crit }}$. We find, in general, that the uncertainty in $L_{\text {crit }}$ is small enough that very few cores change their membership status when the value is shifted to longer or shorter values. Appendix B.1 presents a thorough examination of the effect of different $L_{\text {crit }}$ values on the clusters identified and subsequent analysis results.

We initially calculated $L_{\text {crit }}$ based on the MST structure across the entire Orion A cloud, which gives a value of $0.22 \pm 0.03 \mathrm{pc}$. A visual examination of the resulting clusters, however, showed that this value of $L_{\text {crit }}$ did not successfully identify some visually obvious clusters, especially in the south where some clusters were fragmented or had too few members to be significant. The ISF and its immediate surroundings contain approximately as many dense cores as the rest of the cloud combined, which biased the critical length of the entire cloud toward a smaller value. We removed the mutual bias that ISF and non-ISF cores had on each other by separating cores that were contiguous with a part of the large ISF cluster in the initial MST (as defined by $L_{\text {crit }}=0.22 \mathrm{pc}$ ), from those that were not. We then created a MST and branch length distribution for each sub-population separately. The $L_{\text {crit }}$ for non-ISF cores was $0.36 \mathrm{pc} \pm 0.03 \mathrm{pc}$, while the $L_{\text {crit }}$ for ISF cores was $0.14 \pm 0.01 \mathrm{pc}$.

We identified clusters for the entire cloud using both the ISF and non-ISF $L_{\text {crit }}$ values. Using the larger $L_{\text {crit }}$ value of $0.36 \mathrm{pc}$ produced a set of clusters well-matched to visually apparent clusters in the southern part of Orion A as expected, but also resulted in reasonable clustering in the northern part as well. The ISF was classified as one large cluster, which is reasonable given that it is a visually distinct, single structure in the molecular cloud. Applying the smaller, ISF-derived $L_{\text {crit }}$ of 0.14 pc yielded only the most compact clusters being identified, along with fragments of what are obviously larger structures being picked out as individual clusters. Even the ISF, from which this value was produced, was not divided up in a visually reasonable manner. We therefore decided to use the higher $L_{\text {crit }}$ value of $0.36 \mathrm{pc}$ for the remainder of the analysis on the entire Orion A cloud. Appendix B.1 examines the influence of our choice in $L_{\text {crit }}$ on the the results of our analysis and shows minimal variation within a wide range of choices in $L_{\text {crit }}$. These tests demonstrate that even if we were to find a scheme for determining $L_{\text {crit }}$ that better handled the multiple clustering scales within Orion A, the results from our analysis would be largely unchanged.

\subsection{Physical Characteristics of Clusters}

After the initial cluster identification, we imposed a minimum membership criterion of more than 10 cores to be classified as a cluster, which is the same as the threshold applied in KM11 and K16; Appendix B.2 shows that our final results vary little with other minimum cluster sizes. In total, 11 clusters were identified. Individual clusters can be seen in Figures 4 and 5, while their physical properties are listed in Table 1 . The cluster properties in Table 1 include the number of 

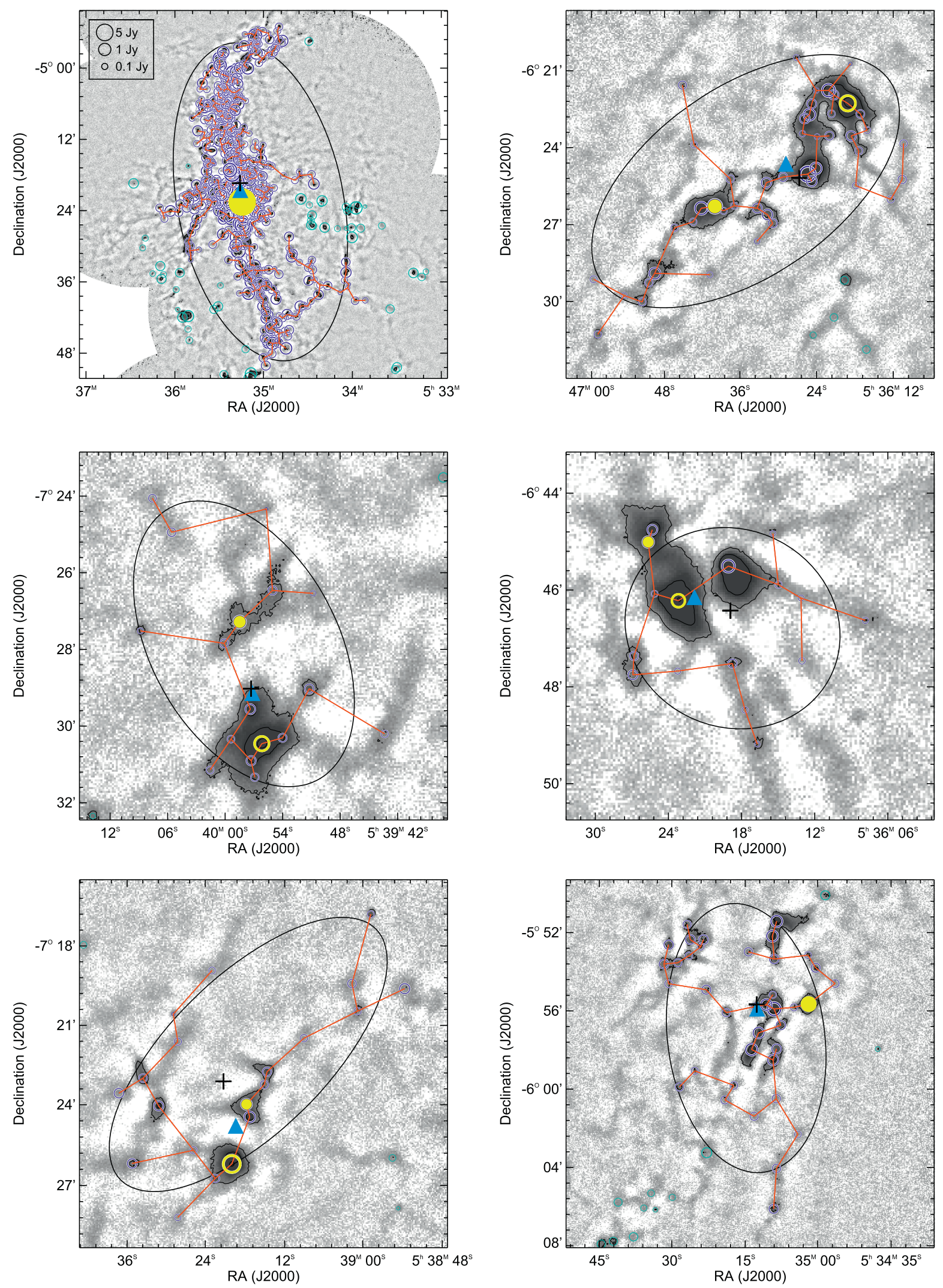

Figure 4. MST-based clusters in Orion A. The grayscale shows the SCUBA-2 $850 \mu \mathrm{m}$ emission, with black contours at $0.02,0.1$, and $0.5 \mathrm{Jy}$ arcsec ${ }^{-2}$. The dark/light blue circles show cluster/non-cluster members identified using getsources, with the circle size scaling with the total flux density. Red lines show the MST structure and the plus sign shows the cluster center. The black ellipse shows a fit to the cluster perimeter (see Section 4.2). The highest flux density cluster member is shown by the open yellow circle, and the highest flux density starless core member with the filled yellow circle (in some cases, the highest flux density cluster member is starless). The center of flux density of each cluster is also shown by the large blue triangle, and generally lies closer to the highest flux density cluster member than the median position does. This figure shows clusters 1 through 6 (top left to bottom right). 

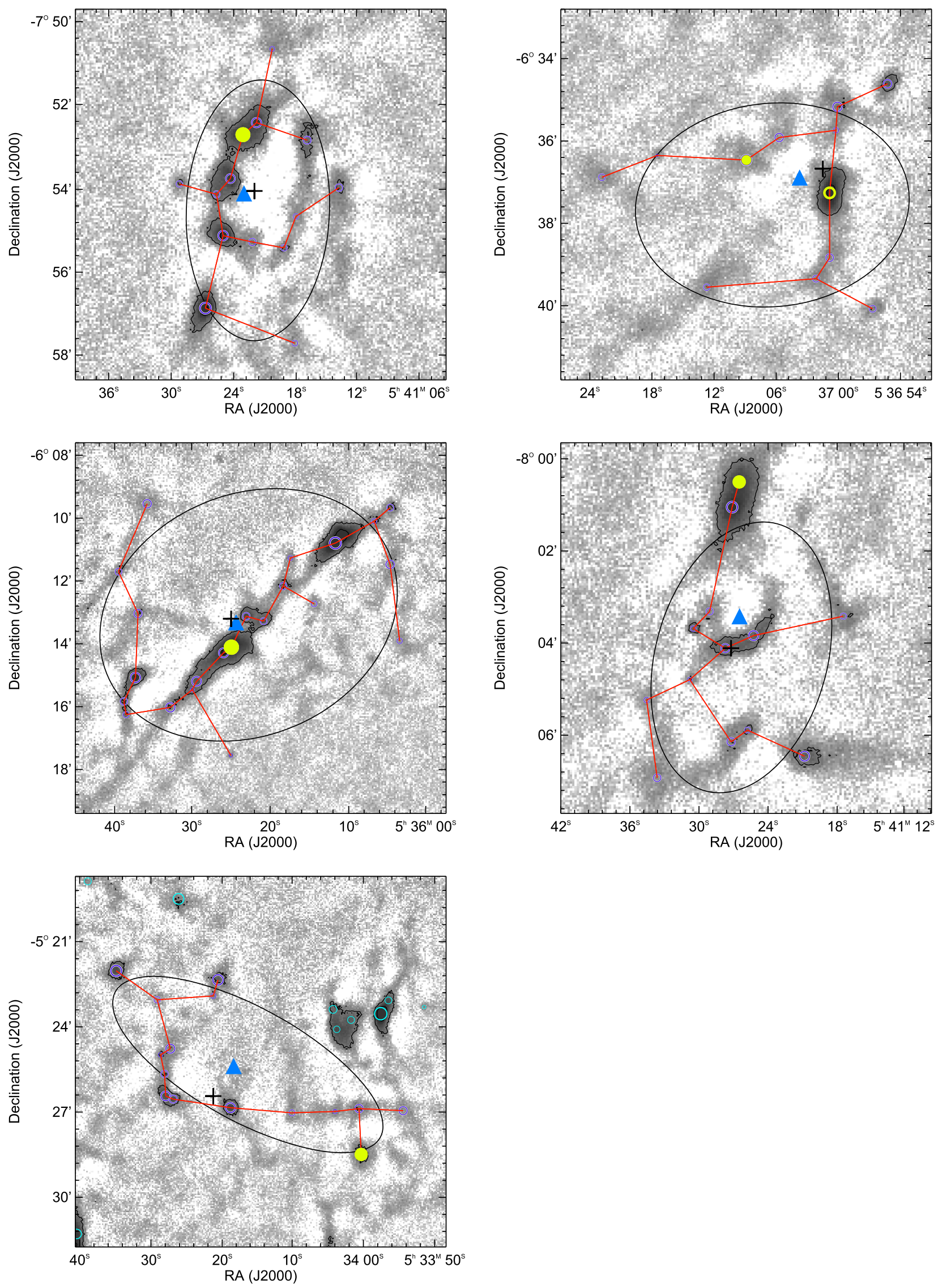

Figure 5. Remaining five clusters in Orion A. See Figure 4 for the plotting conventions used. This figure shows clusters 7 through 11 (top left to bottom). 
Table 1

Properties of MST-based Clusters

\begin{tabular}{|c|c|c|c|c|c|c|c|c|c|c|c|c|c|}
\hline Index & $\begin{array}{l}\text { R.A. }^{\mathrm{a}} \\
(\mathrm{J} 2000)\end{array}$ & $\begin{array}{l}\text { decl. }^{\mathrm{a}} \\
(\mathrm{J} 2000)\end{array}$ & $N^{\mathrm{a}}$ & $\begin{array}{l}S_{\mathrm{rat}}^{\mathrm{b}} \\
(\mathrm{Jy})\end{array}$ & $\begin{array}{c}S_{\text {med }}^{\mathrm{b}} \\
(\mathrm{Jy})\end{array}$ & $\begin{array}{l}O_{\text {rat }}{ }^{\mathrm{c}} \\
\text { (pc) }\end{array}$ & $\begin{array}{c}O_{\text {med }}{ }^{c} \\
(\mathrm{pc})\end{array}$ & $\begin{array}{l}\text { Proto. }^{\mathrm{d}} \\
\text { Frac. }\end{array}$ & $\begin{array}{c}\text { Aspect }^{\mathrm{e}} \\
\text { Ratio }\end{array}$ & $\begin{array}{c}\text { Maj. } \\
(\mathrm{pc})\end{array}$ & $\begin{array}{c}\operatorname{Min}^{\mathrm{e}} \\
(\mathrm{pc})\end{array}$ & $\begin{array}{c}\delta \text { R.A. } \\
\left(^{(\prime)}\right.\end{array}$ & $\begin{array}{c}\delta \text { Decl. }^{\mathrm{e}} \\
\left.{ }^{\prime \prime}\right)\end{array}$ \\
\hline 1 & $5: 35: 15.80$ & $-5: 19: 19.20$ & 462 & 435.98 & 0.26 & 0.29 & 1.43 & 0.06 & 1.95 & 3.56 & 1.83 & 206.5 & 188.6 \\
\hline 2 & $5: 36: 26.55$ & $-6: 25: 07.74$ & 46 & 9.47 & 0.19 & 1.00 & 0.45 & 0.09 & 1.87 & 0.90 & 0.48 & -126.6 & 7.4 \\
\hline 4 & $5: 36: 18.74$ & $-6: 46: 21.70$ & 16 & 5.11 & 0.12 & 1.21 & 0.24 & 0.19 & 1.10 & 0.29 & 0.27 & 2.6 & 21.9 \\
\hline 5 & $5: 39: 21.11$ & $-7: 23: 03.23$ & 20 & 2.51 & 0.21 & 0.36 & 0.45 & 0.25 & 2.30 & 0.88 & 0.38 & 56.6 & -60.7 \\
\hline 6 & $5: 35: 12.44$ & $-5: 55: 37.56$ & 38 & 6.86 & 0.22 & 0.64 & 0.54 & 0.08 & 1.72 & 0.91 & 0.52 & -32.4 & 103.2 \\
\hline 9 & $5: 36: 24.78$ & $-6: 13: 09.09$ & 22 & 8.43 & 0.14 & 0.25 & 0.47 & 0.05 & 1.28 & 0.64 & 0.50 & 33.6 & -7.3 \\
\hline 10 & $5: 41: 27.00$ & $-8: 04: 04.47$ & 13 & 3.68 & 0.16 & 1.78 & 0.27 & 0.23 & 1.61 & 0.39 & 0.24 & 13.5 & 12.0 \\
\hline 11 & $5: 34: 21.02$ & $-5: 26: 23.60$ & 15 & 2.78 & 0.20 & 1.58 & 0.46 & 0.00 & 2.67 & 0.70 & 0.26 & 73.0 & -66.7 \\
\hline
\end{tabular}

Notes.

a The cluster center, measured as the median position of all cluster members, and the number of cluster members.

b The flux ratio and median total flux of cores within the cluster.

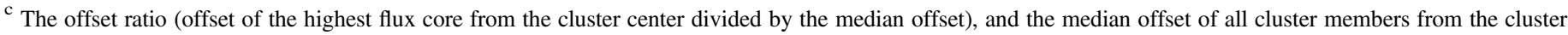
center.

${ }^{\mathrm{d}}$ The fraction of protostellar cores in the cluster.

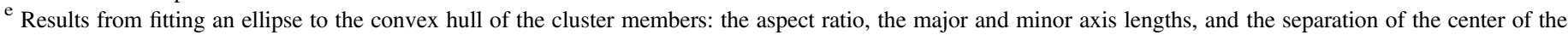
ellipse from the cluster center.

members, the location of the cluster center, the fraction of members classified as protostars, and the aspect ratio of the cluster, in addition to several quantities introduced in Section 4.4 below. One of the clusters (\#11) is listed as having a protostellar fraction of precisely zero in Table 1 . We note that one of the cluster members is in the vicinity of a protostar, but the protostar-core separation is greater than our classification criterion.

We follow KM11 and K16 and define the cluster center as the median position of cluster members. For few-member systems, the median position is more robust to a single outlying member influencing the center than the mean position. Furthermore, we can better prevent undue bias in our later measurement of mass segregation by not adopting the center of mass (or center of flux density) position for the cluster center. Figures 4 and 5 also show both the median position and center of flux location for each cluster, illustrating that the latter tends to be closer to the highest flux cluster member.

\subsection{Elongations}

Following G09, a two-dimensional perimeter was determined for each cluster by forming a convex hull, which is a polygon where all interior angles are less than $180^{\circ}$. An ellipse was fitted to this perimeter to estimate the aspect ratio of each cluster. ${ }^{12}$ As can be seen in Table 1, as well as Figures 4 and 5, many of the clusters are noticeably elongated. We ran some simple checks to determine whether or not these elongations were statistically significant. We created 10,000 synthetic clusters each with 10 , 15 , and 25 members in a random uniform distribution over a 2D circular region. We then ran the same convex hull and ellipsefitting routine and compared the aspect ratios measured for the observed and synthetic clusters. The two were notably different: a two-sample Kolmogorov-Smirnov (KS) test yields a probability of between $1 \%$ and $2 \%$ that the observed cluster aspect ratios are drawn from the same population as the synthetic cluster aspect ratios. We also ran a similar test using a more

\footnotetext{
12 http://www.idlcoyote.com/ip_tips/fit_ellipse.html
}

centrally concentrated distribution for the synthetic clusters (positions randomly distributed equally in radial and angular directions from the cluster center). With this second set of synthetic clusters, we find an even smaller consistency with the observations, with two-sample KS probabilities of the two sets of aspect ratios being drawn from the same sample of $0.4 \%$ and lower for clusters of 15 or more members. Synthetic clusters with 10 members had a higher probability of consistency than the uniformly random distribution, but most of the observed clusters have 15 or more members, so those probabilities are more applicable. The low probability of consistency between the observed cluster aspect ratios and those generated by circularly symmetric random distributions suggests that the elongation seen in the observed clusters is real.

Elongated clusters are perhaps not surprising at the dense core stage. Dense cores appear to be strongly associated with filaments (e.g., André et al. 2014, p. 27), which would tend to fragment to produce long chains of cores. Clusters may form preferentially at the intersection of several filaments (e.g., Myers 2009; Schneider et al. 2012), but depending on the geometry and star-formation density in each filament, such origins could still easily lead to initially non-round dense core clusters.

\subsection{Offset Ratio}

Following KM11 and K16, we use the location of the highest flux density core in each MST-based cluster to look for indications of whether this core is located randomly or if it is preferentially located toward the cluster center. We first measure the radial offset of each cluster member, i.e., the separation of each core from the cluster center. We then compare the offset of the highest flux density core to the median offset of all cluster members $\left(O_{\text {rat }}\right.$ in Table 1$)$, i.e., the offset ratio. Offset ratio values less than one indicate that the most massive member is located close to the center of the cluster, while values larger than one indicate that the most massive member is in the cluster outskirts. For a very rough indication of the relative importance of gravity from the most 

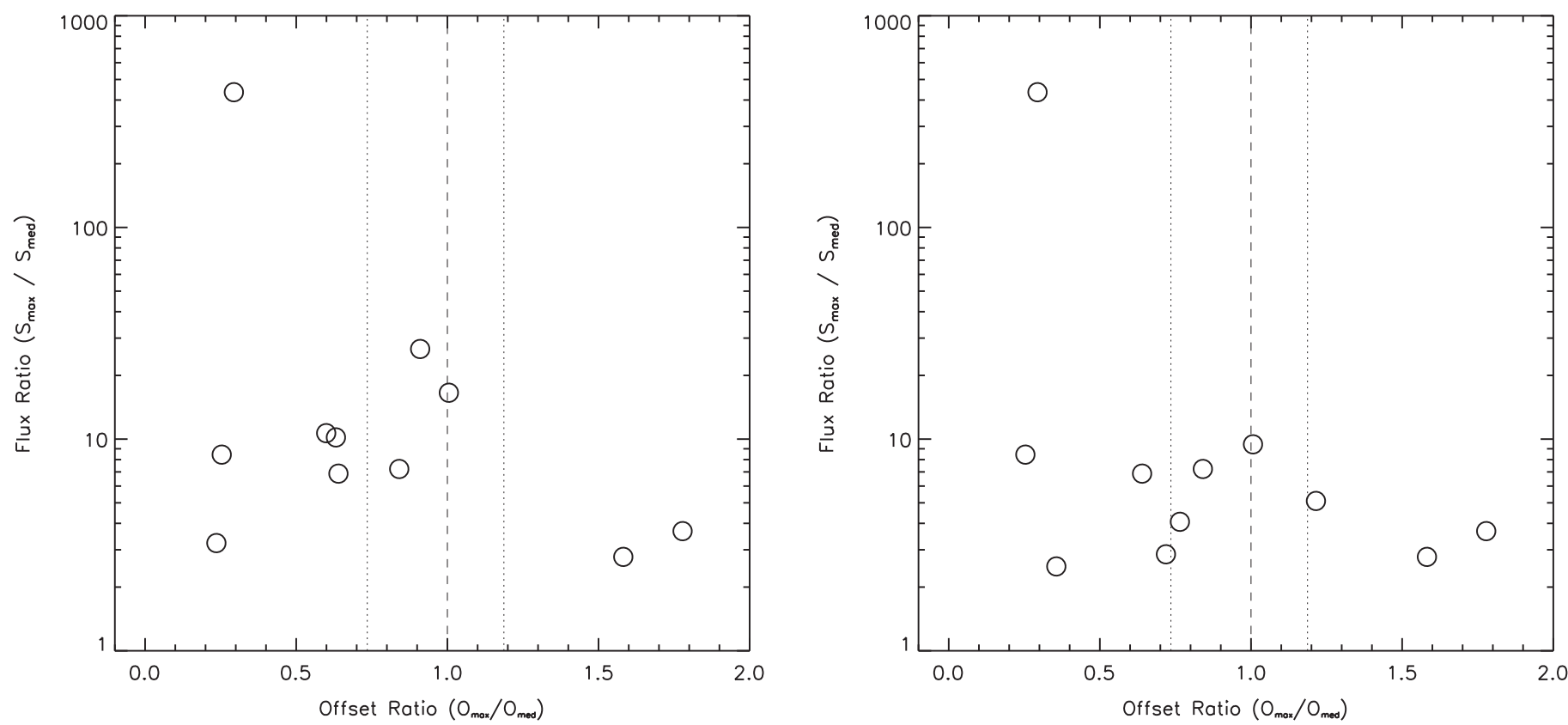

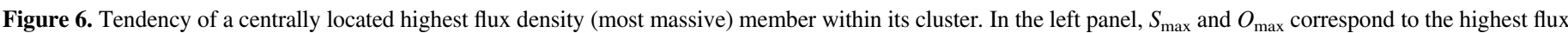

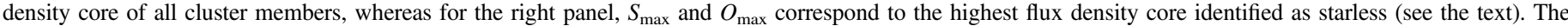

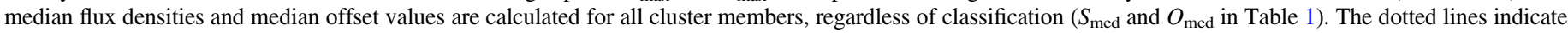

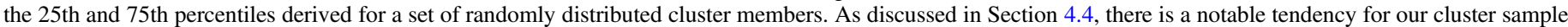
to have offset ratios below one (i.e., lying to the left of the dashed line).

massive cluster member, we calculated the ratio of the flux density of the most massive cluster member to the median cluster member flux density $\left(S_{\text {rat }}\right.$ in Table 1$)$, the flux density ratio. (Recall that under our assumption of a constant conversion factor between flux density and mass, the ratio of flux densities and masses are identical.) Flux ratios near one indicate that all cluster members have similar flux densities, while clusters with flux density ratios much greater than one indicate a large range of flux densities. Higher flux density ratios are also usually indicative of the presence of higher flux density cores. The left panel of Figure 6 shows the flux density and offset ratios of each cluster.

Figure 6 shows that most clusters have centrally located highest flux density (most massive) cores. We ran some tests to check whether or not our observed distribution of offset ratios are consistent with a randomly located most massive member. We created 10,000 synthetic clusters with 15 members (a typical group size) placed randomly within a uniform, twodimensional circular distribution. Within these clusters, we randomly selected a most massive cluster member and calculated the offset ratio in the same way as the observed clusters. The 25 th and 75 th percentiles of this distribution are shown in Figure 6. A two-sample KS test was used to compare the observed offset ratios with the offset ratios from the random distributions. The probability that the random distribution and the observed distribution of offset ratios were drawn from the same sample was $7 \%$, a nearly $2 \sigma$ result. Other synthetic cluster types (e.g., uniform spherical distribution, or different number of cluster members) yield similar distributions of offset ratios, with a roughly equal number of ratios above and below one (see the discussion in KM11), so we would expect similar statistics for such cases. The small sample size of clusters is the driving factor in the statistical significance we find. Including offset ratios of dense cores in clusters in Orion B measured in a similar manner yields an only $3 \%$ probability of consistency with a random distribution (see K16).

To interpret the offset ratios derived as relating to mass segregation, we would need to assume a one-to-one conversion between the flux- and mass-ranking of cores within a cluster (or at least that the core with the highest flux density is also the most massive core). This assumption may not be tenable if cores have a range of temperatures, as discussed in Section 3.2. Following K16, we additionally search for the location of the highest flux density starless core within each cluster, and measure its offset ratio. The rationale here is that the if the highest flux density core only has high flux density due to an elevated temperature, then the highest flux density starless core is the next most likely candidate for the highest mass cluster member. The right-hand panel of Figure 6 shows the offset ratios measured for the highest flux density starless core in each cluster, and these generally follow a similar trend, where most have offset ratios less than one. We note, however, that several of the clusters have a fairly high protostellar core fraction, which limits the number of starless cores available for us to perform this test. In Appendix C, we use Herschel-based temperatures to further test the robustness of our analysis to temperature variations, and demonstrate that the most massive cluster member is nearly always the same as the highest flux cluster member.

\section{MASS SEGREGATION AND SURFACE DENSITY}

In Section 4.4, we found evidence of a preferential central location of the highest flux density/most massive core in each MST-based cluster, suggesting that there is some degree of mass segregation present in these systems. We can also search for mass segregation in Orion A using a second, completely independent method. This method is the $S-\Sigma$ method from (Maschberger \& Clarke 2011), where we have replaced their 

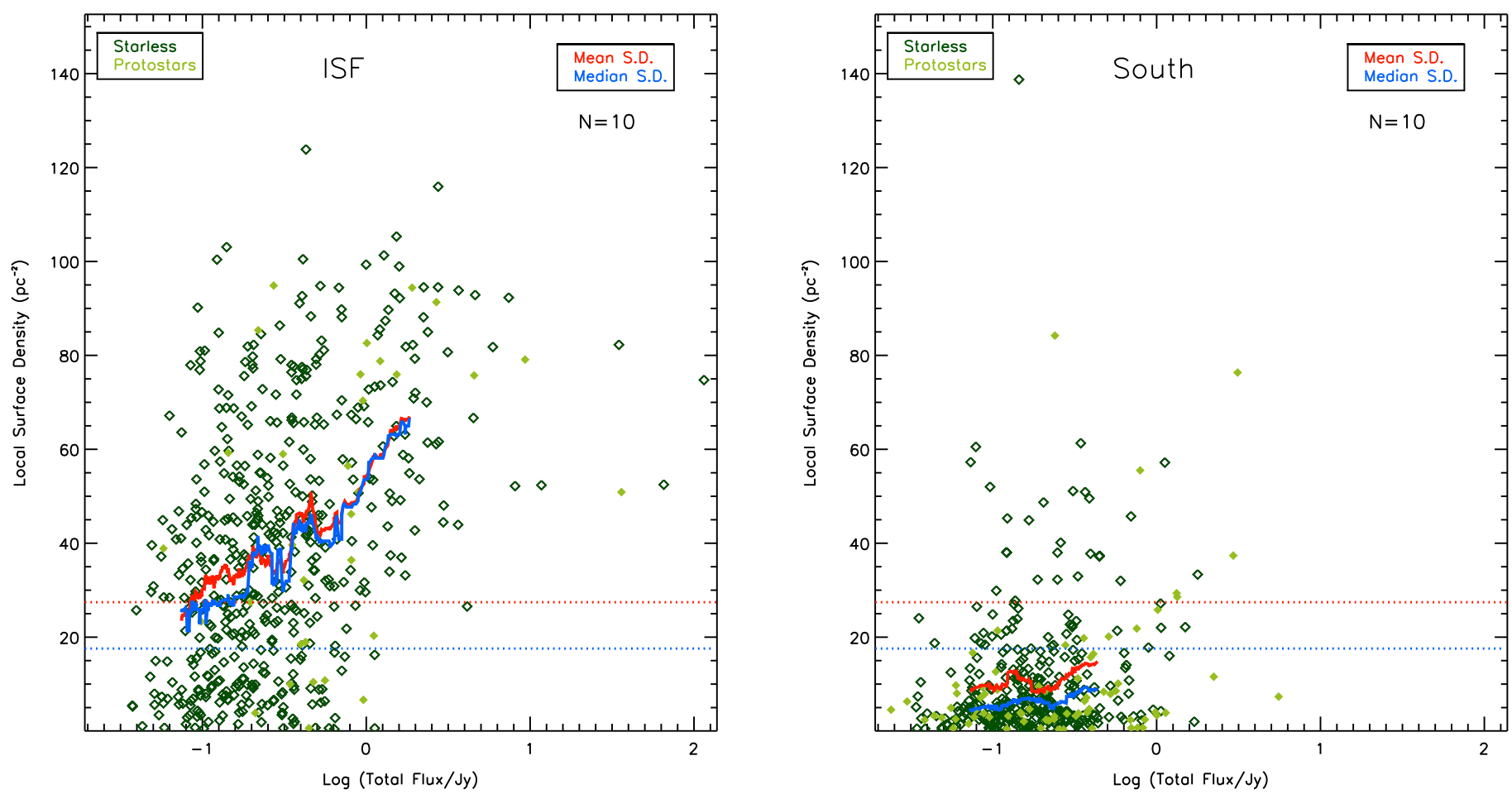

Figure 7. Core-core surface density, as derived from the 10 nearest neighbors (NN10), as a function of core flux density. The left-hand plot shows dense cores in the ISF (having decl. above approximately $-5: 50: 25$ ), while the right-hand plot shows dense cores south of the ISF. Starless cores are shown as dark open diamonds, while protostellar cores are shown as the light filled diamonds. The running mean and median values for the starless cores are shown by the red and blue lines, respectively, calculated by binning 30 values on either side of each point. Both lines highlight the general trend of a higher surface density of cores around higher flux density cores. The dotted horizontal lines show the global mean (red) and median (blue) values.

mass $M$ by the core total flux density $S$. The $S-\Sigma$ method uses the local core-core surface density as an indicator of clustering, and compares the mass (total flux density) of cores to their core-core surface density to look for trends. In the case of a single cluster, mass segregation would clearly be visible as higher mass cores being found at higher core-core surface densities. In systems with multiple sub-clustered populations, mass segregation should still be visible as a tendency for higher mass cores at higher core-core surface densities; though, a large scatter in individual values might be expected. The $S-\Sigma$ measurement is completely independent of any definition of individual clusters, making it an ideal complement to the MSTbased cluster analysis in Section 4.

We calculate the surface density around each core by determining the circular radius needed to encompass a total of $N$ cores, i.e.,

$$
\sigma_{N}=\frac{N}{\pi r_{N}^{2}},
$$

where $\sigma$ is the core-core surface density, and $r$ is the circular radius encompassing $N$ cores. The fractional uncertainty associated with this surface density estimate scales as $N^{-0.5}$ (Casertano \& Hut 1985; Gutermuth et al. 2009). In our analysis, similar to K16, we used both the closest 5 cores (NN5) and the closest 10 cores (NN10), and find similar results with both. In Figure 7, we show the NN10 core-core surface densities plotted against each core's total flux density. For this figure, we separately consider cores in the vicinity of the ISF and further south in Orion A to ensure that our results are not biased by behavior present in only one of the two core populations. Both panels of Figure 7 show a clear trend highlighted by the red and blue co-moving mean and median values, that the total core flux density tends to be higher in regions with a higher surface density of neighboring cores; though, the slope of the co-moving mean and median values differ between the ISF and the south. As with our MST analysis in Section 4, we note that protostellar cores may have systematically higher total flux densities than starless cores for the same intrinsic mass, due to the typically higher temperature of the former population. We handle this potential bias in our analysis by analyzing the starless cores and protostellar cores separately, as well as jointly. In our statistical tests below, the protostellar cores represent the smallest population, and therefore show the poorest statistical significance in the results reported. The co-moving mean and median lines shown in Figure 7 for illustrative purposes only are based on the starless core population.

We employ a two-sample KS test to evaluate the significance of the apparent trend of higher flux density cores being found in higher surface density environments. First, we compare the core-core surface densities for the higher flux density half of the cores with the core-core surface densities for the lower flux density half of the cores to see whether or not the two distributions of surface densities are consistent. We also run a similar test using the surface densities associated with the cores with the lowest and highest third of the flux densities. In both of these cases, and using either of the NN5 and NN10 derived surface densities, and running the analysis across all of Orion A, or examining the ISF and the south separately, the probability that the highest and lowest flux density cores have surface densities drawn from the same parent population is less than $10^{-7} \%$ for either the starless cores alone, or for both the 
starless and protostellar cores analyzed together. For the protostellar cores alone, the smaller population size, especially when split between the ISF and the south, lead to somewhat less significant results: the probability is less than $5 \%$ in all cases, with many of the tests giving probabilities of less than $1 \%$.

We also ran a Mann-Whitney test, which compares the ranking of values within two samples to determine if one has typically larger values. As expected from the clear positive slope seen in the co-moving mean and median lines indicated on Figure 7, the Mann-Whitney test shows a strong probability of higher flux density cores inhabiting higher surface density environments. For the starless cores, or starless and protostellar core samples, we find probabilities of $10^{-9} \%$ or less that the higher flux density cores do not inhabit higher surface density environments, i.e., nearly $100 \%$ likelihood that higher flux density cores do inhabit higher surface density environments. The one exception to this result is the probability measured for the starless core population in the south, which has a value of $2 \%$ or less, which still implies a $98 \%$ or higher likelihood of higher flux density cores inhabiting higher surface density environments. For the protostellar core populations, all probabilities are below 7\%, with all but two tests giving probabilities below $1 \%$. We therefore conclude that the dense cores in Orion A show strong evidence for a tendency for high flux density cores to be located in high core-core surface density environments.

In Appendix $\mathrm{C}$, we find similar results using instead estimated masses based on Herschel-derived temperatures for the dense cores.

\section{DISCUSSION}

\subsection{Cluster Identification}

Identifying clusters of cores in molecular clouds, which tend to have a hierarchical structures, is difficult at the best of times (see, for example, the discussion of hierarchical structure within Orion in Takahashi et al. 2013). This situation is complicated further in the analysis of dense cores, since the identification of cores is also challenging. We examine the uncertainty in our main results arising from these complications in a variety of ways. In Appendix A, we examine the impact of differing core identification algorithms. We first compare the core catalog used in our main analysis (getsources) with another independent technique (FellWalker) in Appendix A.1.3, and find that the main difference is in how complex emission structures are divided (rather than whether or not emission is associated with a core at all). We also compare with the core catalogs from other SCUBA-2 analysis, namely Mairs et al. (2016) in the south (see Appendix A.1.4) and Salji et al. (2015a) in the north (see Appendix A.1.5), both of which use different core identification techniques than our analysis. Both of the island and fragment catalogs of Mairs et al. (2016) similarly correspond well with our core catalog, while there is poorer correspondence with Salji et al. (2015a) driven largely by constraints in core properties those authors imposed.

To determine whether or not the core identification technique influences our results, we re-ran all of the analyses presented in the paper using instead the FellWalker-based core catalog. While the numbers and properties of the cores vary significantly (919 with getsources and 773 with FellWalker), the conclusions drawn from the clustering analysis remain largely the same.

For our results relying on the MST analysis (Section 4.4), we additionally test the effects of uncertainty in $L_{\text {crit }}$ and the choice in minimum cluster size on the clusters identified, as well as the results we derive based on the MST clusters. These tests are shown in Appendix B, and here too, we find that our overall conclusions are similar.

These comparisons and tests all suggest that the results of our analysis are consistent, regardless of the core extraction method and parameters used in the MST analysis.

\subsection{Smaller-scale Fragmentation}

Another effect that should be considered is how the dense cores we observe might fragment on scales smaller than we are able to resolve with the JCMT. The northern portion of the ISF was recently observed with ALMA by Kainulainen et al. (2016), who identified 40 sources with $3^{\prime \prime}$ resolution. They found evidence of clustering on all scales examined, with no preferential length scale associated with the clustering; instead, clustering became increasingly evident down to the minimum size probed by their resolution. Much of the clustering detected by Kainulainen et al. (2016) is on scales below that observable with our SCUBA-2 data: they report detecting significant clustering at scales of $17000 \mathrm{au}$ and smaller $(\leqslant 0.08 \mathrm{pc})$, particularly for their starless sources, while the SCUBA-2 $850 \mu \mathrm{m}$ beam of 14 ." 6 corresponds to a scale of $\sim 0.03 \mathrm{pc}$.

In the protostellar regime, Kounkel et al. (2016) searched for binaries and higher order stellar systems with separations of about 100-1000 au across Orion A using Hubble observations. They find a companion fraction of about $14 \%$ for the protostellar sources, a similar fraction to that obtained earlier in Taurus. They furthermore note that the companion fraction appears to be somewhat higher for protostars living in high stellar density regions of the cloud, with a roughly $50 \%$ increase in the companion fraction for protostars in $\Sigma>45$ $\mathrm{pc}^{-2}$ environments versus those in lower surface density environments. Based on the Kounkel et al. (2016) results, we might therefore expect that the dense cores in the ISF will fragment more than those further south in L1641. The degree of extra fragmentation at higher local source surface densities measured, however, does not appear to be significant enough to invalidate our assumption that the more massive protostars form out of the more massive dense cores, i.e., there is no indication that the most massive dense cores form only a large number of low-mass protostars.

Therefore, although small-scale fragmentation certainly exists in Orion $\mathrm{A}$, the existing data does not suggest that our results would qualitatively change if our analysis were performed using higher resolution data.

\subsection{Interpretation}

One of the main results of our analysis is that most of the clusters of dense cores in Orion A appear to have some degree of mass segregation already. We investigated this using two independent techniques. First, we used an MST to identify clusters, which we found have tentative evidence that the highest flux density (most massive) cluster member was preferentially found toward the cluster center. Second, we applied the $S-\Sigma$ technique, which uses the core-core surface density as a proxy for clustering and found a trend 
between core flux density and local core-core surface density. Measurements of mass segregation are often controversial, in part due to differences in the type of system (e.g., single cluster or multiple subclusters) and the size scale that is appropriate to analyze, as discussed in K16 and also Girichidis et al. (2012). The two methods we adopted in our analysis both allow for the possibility of multiple clusters or sub-clustered systems within the region analyzed, and both are also effectively localized measures.

A third popular method that we did not use is the $\lambda_{\text {MST }}$ technique pioneered by Allison et al. (2009b). The $\lambda_{\text {MST }}$ method performs very well in tests of searching for mass segregation within a single cluster (Parker \& Goodwin 2015), but tends instead to measure features in the large-scale structure when applied to wide areas containing multiple subclusters, such as the Taurus molecular cloud, as discussed in K16. Like Taurus, the Orion A molecular cloud is a multi-parsec structure, which is not best represented as a single star-forming cluster where cluster members have the potential to interact with each other. In Orion $\mathrm{A}$, the typical denser gas velocity dispersion is $\sim 1$ to $5 \mathrm{~km} \mathrm{~s}^{-1}$ based on $\mathrm{C}^{18} \mathrm{O}(3-2)$ HARP observations (Buckle et al. 2012), resulting in a crossing time along the narrow axis of Orion A ( $\sim 5 \mathrm{pc}$ wide in our SCUBA-2 map) of $1 \mathrm{Myr}$, which is significantly larger than the typical estimated dense core lifetime of several tenths of a megayear (e.g., Kirk et al. 2005; Hatchell et al. 2007; Enoch et al. 2008; Könyves et al. 2015). The $\lambda_{\text {MST }}$ method is therefore expected to trace the large-scale assembly of lower density cloud material, while the MST and $S-\Sigma$ methods allow for substructure within Orion A to be separately considered.

Using the MST method, we find that most of the clusters identified have a centrally located most massive member (i.e., offset ratios less than one). The offset ratios measured differ from those expected from a randomly located most massive member at the $93 \%$ confidence level, with the relatively low statistical significance driven in part by the small sample size. Including the dense core clusters analyzed in Orion B in a similar manner by K16, where the distribution of offset ratios are similar (roughly the same proportion of offset ratios below and above one), the result becomes more significant (97\% likelihood of non-random locations). Using the $S-\Sigma$ technique, we find that more massive cores tend to live in higher core-core surface density environments at well above a 99.99\% significance level. The statistical significance of this measure is much greater in part because every core contributes a measurement, rather than using only one measurement per cluster.

Our results, showing that more massive dense cores tend to occupy more clustered environments, may suggest that the centers of clusters provide a favorable environment in which more massive protostars can form (see also Myers 2011). In Orion A, there is already some evidence that highly clustered environments favor the formation of more massive stars: Hsu et al. (2012) and Hsu et al. (2013) compare the young stellar populations in the ISF and L1641 (southern Orion A), and conclude that there is some evidence for a deficit of the most massive stars (O- and early-B-type) in L1641 compared to the ISF. Our results suggest that within southern Orion A, there will be a relative over-abundance of the most massive protostars within highly clustered zones and a relative dearth of the most massive protostars in the sparser regions of the south. These relative abundances are consistent with the Hsu et al. results, so long as the overall fraction of massive protostars in southern Orion A is lower than in the ISF, a scenario that seems reasonable in the context of our results.

Tying our mass segregation results to star-formation models, such as turbulent core accretion (McKee \& Tan 2003) and competitive accretion (Bonnell et al. 2001) is not yet possible. Predictions from each model on the expected spatial distribution of dense core masses are needed first. Naively, the competitive accretion model might be expected to be a better fit, since young protostars are expected to be mass segregated in that model. Unlike the turbulent core accretion model, however, the majority of material accreted by protostars does not originate in the protostar's natal core in the competitive accretion model (see, e.g., Tan et al. 2014, p. 149); therefore, it is unclear whether dense cores should show signs of mass segregation or if segregation should only be present in the protostars. The idea of cluster centers providing a favorable accretion environment is, however, consistent with other lines of evidence suggesting that protostellar clusters form with some degree of primordial mass segregation. As discussed in K16, several recent studies of young protostellar clusters find evidence of centrally located more massive members (e.g., Megeath et al. 2005; Hunter et al. 2006; Kryukova et al. 2012; Elmegreen et al. 2014); though, at least one other study comes to a different conclusion (Hunter et al. 2014). Observations of slightly older protostellar clusters also often show evidence of mass segregation, and estimate that the clusters are too young to be able to explain this through dynamical evolution (e.g., Carpenter et al. 1997; Hillenbrand 1997; Bonnell \& Davies 1998; Stolte et al. 2006; Gennaro et al. 2011; Kirk \& Myers 2011; Davidge 2015); though, this interpretation is somewhat controversial, with other authors finding a lack of evidence of primordial (or sometimes present day) mass segregation (e.g., Allison et al. 2009a, 2010; Parker et al. 2012; Wright et al. 2014). The observations we analyze here show the clustering behavior at an early stage, and it is important to emphasize that the masses, and to a lesser extent, the locations, of the dense cores will continue to evolve beyond the snapshot in time that our observations capture. As noted above, the centers of clusters are thought to provide an environment favorable to higher mass accretion (e.g., Myers 2011), and this has also been observed in some numerical simulations (e.g., Smith et al. 2009), where the most massive protostar accretes material that originated from throughout the cluster rather than only its natal core. While all of the cores are expected to continue to accrete material, those inhabiting the centers of clusters may experience a larger rate of growth than those on the outskirts. The motion between dense cores and their surrounding less dense envelopes is generally small throughout a cluster (typically less than the sound speed, e.g., Walsh et al. 2004; Kirk et al. 2007; Walsh et al. 2007), but the motion is larger when considering the dense core versus lower density ambient cluster material (e.g., Kirk et al. 2010), suggesting accretion of material from larger scales is important.

It is interesting to note that the results of our clustering analysis of dense cores in Orion A so closely mimics the results seen in Orion B (K16), as well as the older, small and sparse protostellar groups analyzed in KM11. Orion is well-known for having one of the higher density and more turbulent environments forming a wider range of stellar masses, than is present within other nearby (Gould Belt) clouds. Nonetheless, 
the southern portion of Orion A already shows a much sparser, looser clustering environment than cores near the ISF, but both parts of Orion A exhibit signs of mass segregation. According to Stutz \& Gould (2016), the ISF has a much steeper gravitational potential well than the material in L1641 further south. They postulate that a strong magnetic field around the ISF is helping to drive cluster-forming instabilities in the gas there, a situation which they propose will eventually propogate further south to L1641. Other regions with smaller gas reservoirs would likely never reach an ISF-like stage of cluster formation. This proposed scenario illustrates the importance of testing core clustering behavior under different initial conditions: though we find consistent results across the two varied environments within Orion A, other molecular clouds could still be different. Extension of a clustering analysis to more quiescent, smaller cluster-forming clouds within the Gould Belt will, therefore, provide an important window into how large a role environment plays in clustering properties at these very early times, versus the role of dynamical evolution during protostellar accretion.

\section{CONCLUSIONS}

The JCMT Gould Belt Survey mapped $\sim 6.1$ square degrees of the Orion A molecular cloud down to cores of several hundredths of a solar mass, allowing for dense cores to be identified in a uniform manner across the highly clustered and active integral shaped filament region in the north, as well as dispersed, more quiescent zones of star formation further south. We analyze the clustering properties of the dense cores across the Orion A molecular cloud, and, using two independent techniques, find evidence of mass segregation. Using MSTs, we find that the highest flux density (likely most massive) dense core within each cluster tends to be centrally located. We also find that regardless of cluster definition, dense cores with higher flux densities tend to be located in regions of higher core-core surface density (the $S-\Sigma$ technique). Both of these results have also been found in the complementary analysis of Orion B by K16. These two directions of analysis suggest that at least in some environments, there may be some level of mass segregation imprinted in cluster-forming regions prior to star formation. Furthermore, the clusters of dense cores identified using MSTs show statistically significant elongations, which we speculate may be tied to the filamentary nature of starforming gas.

The authors thank their anonymous referee for a constructive and thorough report, which improved this paper. The authors wish to recognize and acknowledge the very significant cultural role and reverence that the summit of Maunakea has always had within the indigenous Hawaiian community. We are most fortunate to have the opportunity to conduct observations from this mountain. J.L. and H.K. thank Herzberg Astrophysics at the National Research Council of Canada for making this project possible through their co-op program. The authors thank Peter Martin (U. Toronto) for providing computational support for the getsources calculations used in this analysis. The JCMT has historically been operated by the Joint Astronomy Centre on behalf of the Science and Technology Facilities Council of the United Kingdom, the National Research Council of Canada and the Netherlands Organisation for Scientific Research. Additional funds for the construction of SCUBA-2 were provided by the Canada Foundation for
Innovation. The identification number for the programme under which the SCUBA-2 data used in this paper is MJLSG31. ${ }^{13}$ The authors thank the JCMT staff for their support of the GBS team in data collection and reduction efforts. The Starlink software (Currie et al. 2014) is supported by the East Asian Observatory. These data were reduced using a development version from December 2014 (version 516b455a). This research used the services of the Canadian Advanced Network for Astronomy Research (CANFAR), which in turn is supported by CANARIE, Compute Canada, University of Victoria, the National Research Council of Canada, and the Canadian Space Agency. This research used the facilities of the Canadian Astronomy Data Centre operated by the National Research Council of Canada with the support of the Canadian Space Agency. Figures in this paper were creating using the NASA IDL astronomy library (Landsman 1993) and the Coyote IDL library. ${ }^{14}$

Facility: JCMT (SCUBA-2).

Software: Starlink (Currie et al. 2014), CUPID (Berry et al. 2007), IDL, Getsources (Men'shchikov et al. 2012).

\section{APPENDIX A SOURCE-FINDING ALGORITHMS: GETSOURCES VERSUS FELLWALKER}

\section{A.1. Core Catalogs}

Source identification is a major challenge in submillimeter observations of molecular clouds. Sources tend to be irregularly shaped and are often embedded in large-scale structure, which makes it difficult for source extraction algorithms that attempt to fit sources with Gaussian or power-law profiles. Different extraction algorithms manage these issues in their own way. The getsources algorithm decomposes maps onto a variety of spatial scales and identifies structures on each of those scales to characterize best cores and filaments. In this way, small, faint cores can often be detected alongside brighter cores even in regions of complex emission structures. The FellWalker algorithm, on the other hand, identifies peaks based on local gradients. This has the advantage of not assuming any specific shape for a core, but the disadvantage of not allowing for multiple size-scales of structures to contribute emission to a single pixel. Our implementation of each of these algorithms is discussed below.

\section{A.1.1. Getsources}

The getsources algorithm is a multi-wavelength source extraction algorithm designed for the analysis of Herschel observations ${ }^{15}$ (Men'shchikov et al. 2012). One of the main difficulties with source finding in star-forming regions is that dense cores may have contributions to their observed flux density from any number of sources other than the core itself, such as filaments, large-scale overdensities, and noise. Images are analyzed across a range of spatial scales to separate emission arising from these sources. The algorithm first decomposes an image by convolving it with circular Gaussians of a wide range of sizes ( 2-100 pixels) and subtracting one convolution from the next larger convolution. The Gaussian

\footnotetext{
${ }^{13}$ Note that one scan from Orion B is presently mislabelled in CADC with this project code. Observations taken during science verification across all GBS regions falls under the project code MJLSG22.

${ }^{14}$ http://www.idlcoyote.com/index.html

15 http://www.herschel.fr/cea/gouldbelt/en/getsources/
} 
Table 2

Settings for Getsources

\begin{tabular}{|c|c|c|c|}
\hline Phase $^{\mathrm{a}}$ & Parameter & Value & Description \\
\hline $\mathrm{P}$ & instrument & scuba8/scuba4 & Designates data source (for $850 / 450 \mu \mathrm{m}$ ) \\
\hline $\mathrm{P}+\mathrm{E}$ & beamsize & $14 ! .6 / 9 ! .8$ & Effective beamsize at each wavelength (values from Dempsey et al. 2013) \\
\hline $\mathrm{P}$ & mapmaker & other & Specify non-Herschel software used to create maps \\
\hline $\mathrm{P}$ & pixel & $3^{\prime \prime}$ & Pixel size of input map \\
\hline $\mathrm{P}$ & rotangle & 0 & Rotation of maps east from north \\
\hline $\mathrm{P}$ & cutfact & 0.9 & Used in extraction mask creation; 0.9 is a recommended value \\
\hline
\end{tabular}

Note.

a There are two phases in getsources source-finding: Preparation $(\mathrm{P})$ and Extraction $(\mathrm{E})$.

Table 3

Settings for FellWalker

\begin{tabular}{|c|c|c|}
\hline Parameter & Value & Description \\
\hline rms & 0.469 & rms noise per pixel in the image (in mJy/pixel) \\
\hline FellWalker.AllowEdge & 0 & eliminate cores touching map edge \\
\hline FellWalker.CleanIter & 4 & smooth jagged clump edges \\
\hline FellWalker.FlatSlope & 0.05 & increase minimum gradient for pixels to be assigned to a core \\
\hline FellWalker.FwhmBeam & 3 & beamsize in pixels (underestimated to help include small cores) \\
\hline FellWalker.MaxBad & 0 & eliminate cores lying beside bad pixels \\
\hline FellWalker.MinDip & $3^{*} \mathrm{rms}$ & increase minimum dip required between neighboring cores \\
\hline FellWalker.MinHeight & $2^{*} \mathrm{rms}$ & minimum value for peak of core \\
\hline FellWalker.MinPix & 20 & minimum number of pixels for a core \\
\hline FellWalker.Noise & $1^{*} \mathrm{rms}$ & mininum flux in a pixel assigned to a core \\
\hline
\end{tabular}

sizes used for convolving at different scales are separated by a small factor $(\sim 1.03-1.05)$ to ensure high resolution across the range of decomposed images. Each single-scale decomposition is then cleaned of non-relevant signals, such as noise, by using an iterative algorithm that finds a unique cutoff threshold for significant flux density based on the standard deviation of the single-scale image being cleaned. The clean single-scale images are then combined across all wavelengths used in the extraction. Once cores are identified, their properties are extracted by fitting a two-dimensional, elliptical Gaussian to the core. For more information on the getsources algorithm see Men'shchikov et al. (2012). We list the few source-finding parameters that were not set to the explicit default or single recommended value in Table 2.

The getsources catalog we produced was vetted to remove potentially spurious sources. We first removed sources that had no significant detection at $850 \mu \mathrm{m}$, i.e., sources identified by getsources as having SIG_MONO_850 $<7$, which roughly corresponds to a minimum signal-to-noise ratio $(\mathrm{S} / \mathrm{N})$ of 7 at $850 \mu \mathrm{m}$, were removed (this threshold is the recommended threshold to distinguish between reliable and tentative sources). Cores were not required to have similar significant detection at $450 \mu \mathrm{m}$. Observations at $450 \mu \mathrm{m}$ typically have a lower $\mathrm{S} / \mathrm{N}$ than observations at $850 \mu \mathrm{m}$, so cores that may appear distinct and real at $850 \mu \mathrm{m}$ may not always have detections at $450 \mu \mathrm{m}$. We tested several schemes to eliminate further spurious or suspect sources, and found that the method described below was the most visually successful in retaining real sources while eliminating suspect ones. The approach we adopted was to smooth the $850 \mu$ m map using a 5 pixel boxcar kernel and examine the flux densities of each source. Peaks were excluded from the final catalog if they fell below 2.1 times the locally averaged (i.e., within the extraction ellipse defined by getsources) rms noise per pixel. This criterion removed spurious sources and ensured that dense cores detected in noisy regions were in fact significant sources. (We adopt a similar check for the FellWalker catalog discussed below.) Finally, sources which had peak or total flux density errors greater than the respective measurement, which are flagged as undetectable by getsources, were also removed. This vetting reduced the initial getsources "reliable" catalog of 1178 sources to the final robust core catalog of 919 sources.

\section{A.1.2. FellWalker}

FellWalker (Berry 2015) is a source extraction algorithm developed for the Starlink CUPID software package (Berry et al. 2007). ${ }^{16}$ It works analogously to a hiker attempting to find their way to the top of a peak by following the route of steepest ascent. The algorithm starts a "walk" from each pixel in an image and traces its way to a local maximum by following the steepest increasing flux density gradient. Once the program reaches a local maximum it scans a predefined area around the maximum to see if a higher-valued pixel can be found, and if it finds one, it jumps to that pixel and continues its walk toward the next local maximum. In this manner, FellWalker will eventually find a significant local maximum and this point is labeled as a peak. The pixels of each walk that end at the same peak are attributed to one core. To limit the physical extent of the sources to realistic sizes, parts of a walk that occur along a gradient that is shallower than a certain threshold are not included. There are other input parameters that mitigate the effects of background noise and sources with excessive substructure, as well as determining how significant a peak must be for it to be considered a core. Table 3 summarizes all of the FellWalker parameters that we set to non-default values along with a short description of their purpose. More

\footnotetext{
${ }^{16}$ Available at http://starlink.eao.hawaii.edu/starlink/CUPID
} 

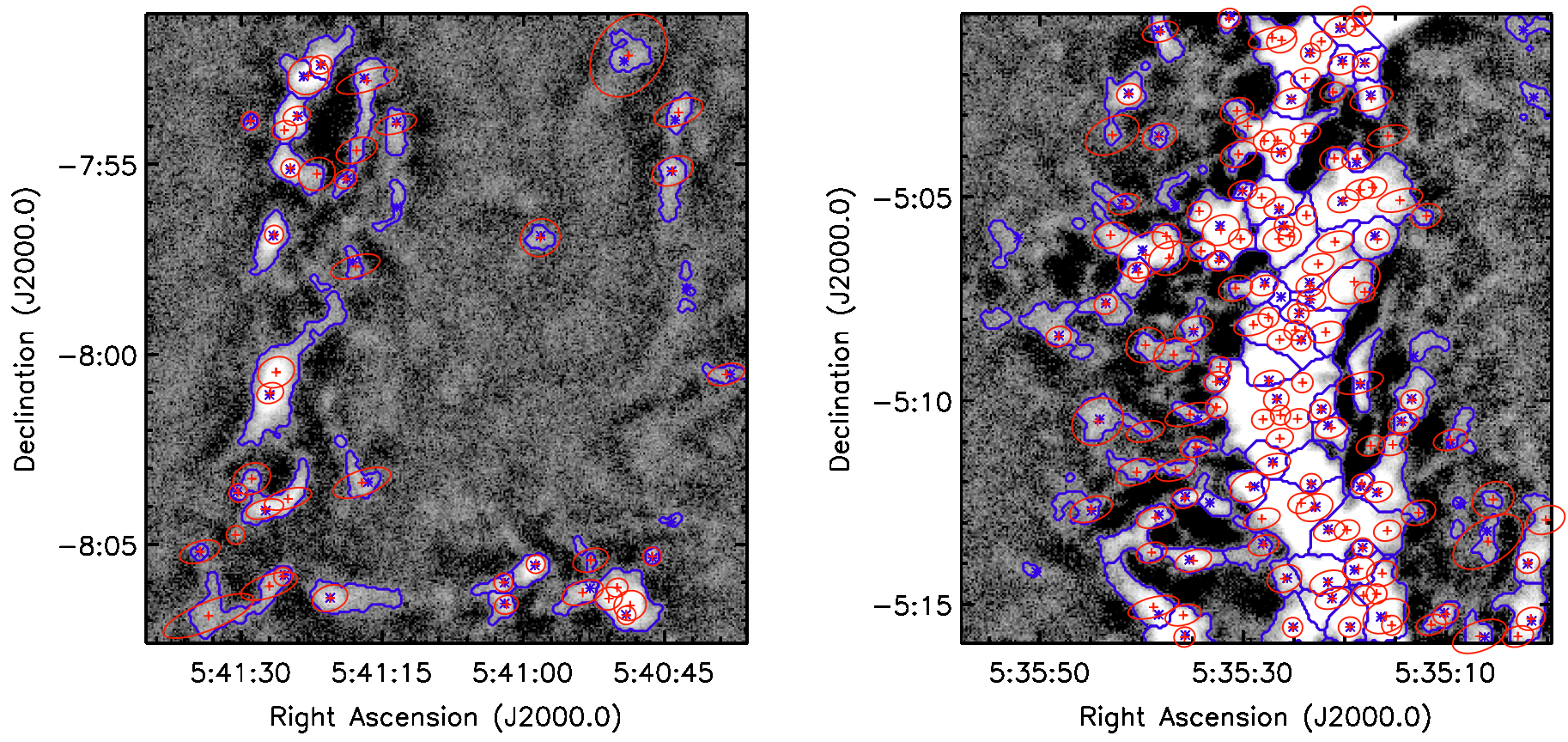

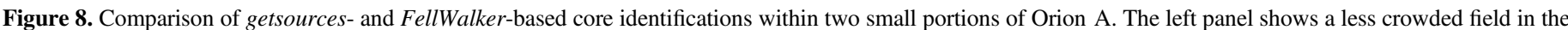

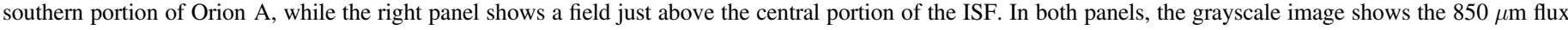

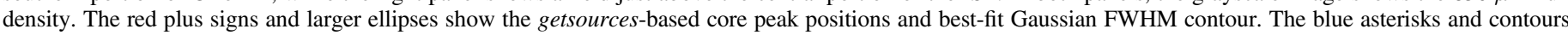
show the FellWalker-based core peak positions and full extents.
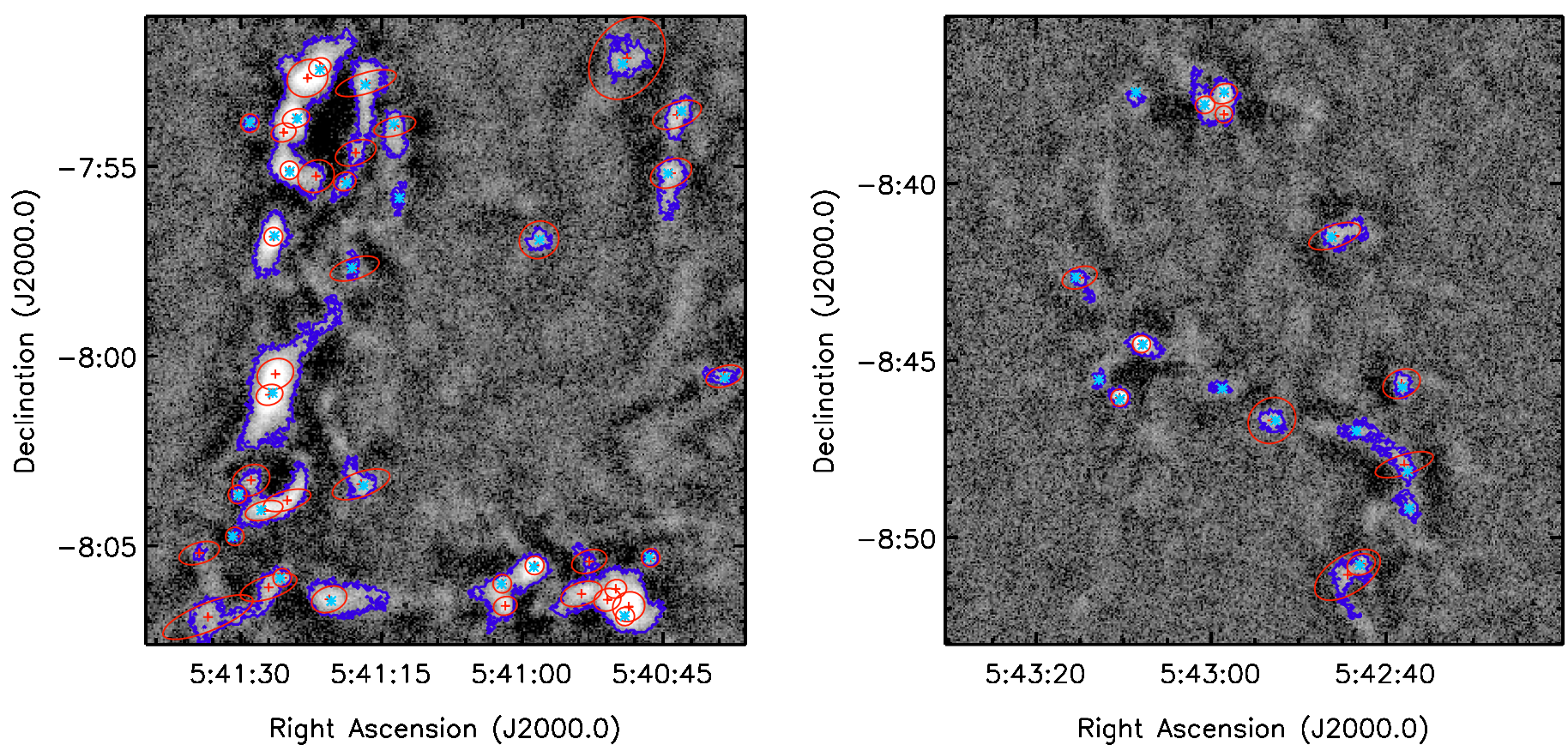

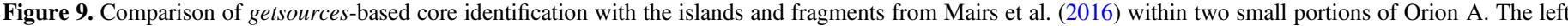

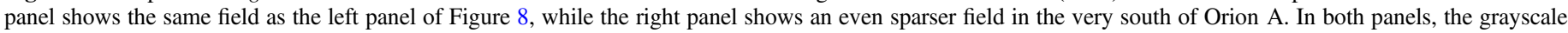

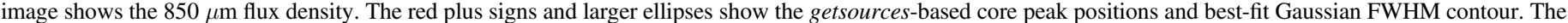

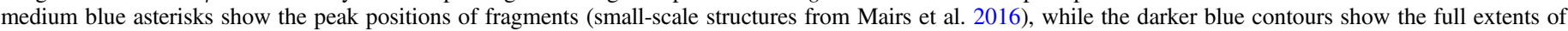
islands (larger scale structures from Mairs et al. 2016).

information about FellWalker and the parameters that can be set is available in Berry (2013).

In our initial core identification, we experimented with a variety of peak flux density thresholds and minimum size requirements. The final settings were selected to include the maximum number of significant cores in the catalog. FellWalker uses a single global noise value to determine whether or not sources are real, which we set to the global average rms noise per pixel, i.e., $0.0042 \mathrm{Jy} \mathrm{arcsec}^{-2}\left(0.469 \mathrm{mJy}_{\mathrm{pixel}}{ }^{-1}\right)$. The minimum height for a core was set to two times this rms noise level. Since the noise level in the mosaic is not constant (in particular, it is higher near the map edges); however, a number of spurious 

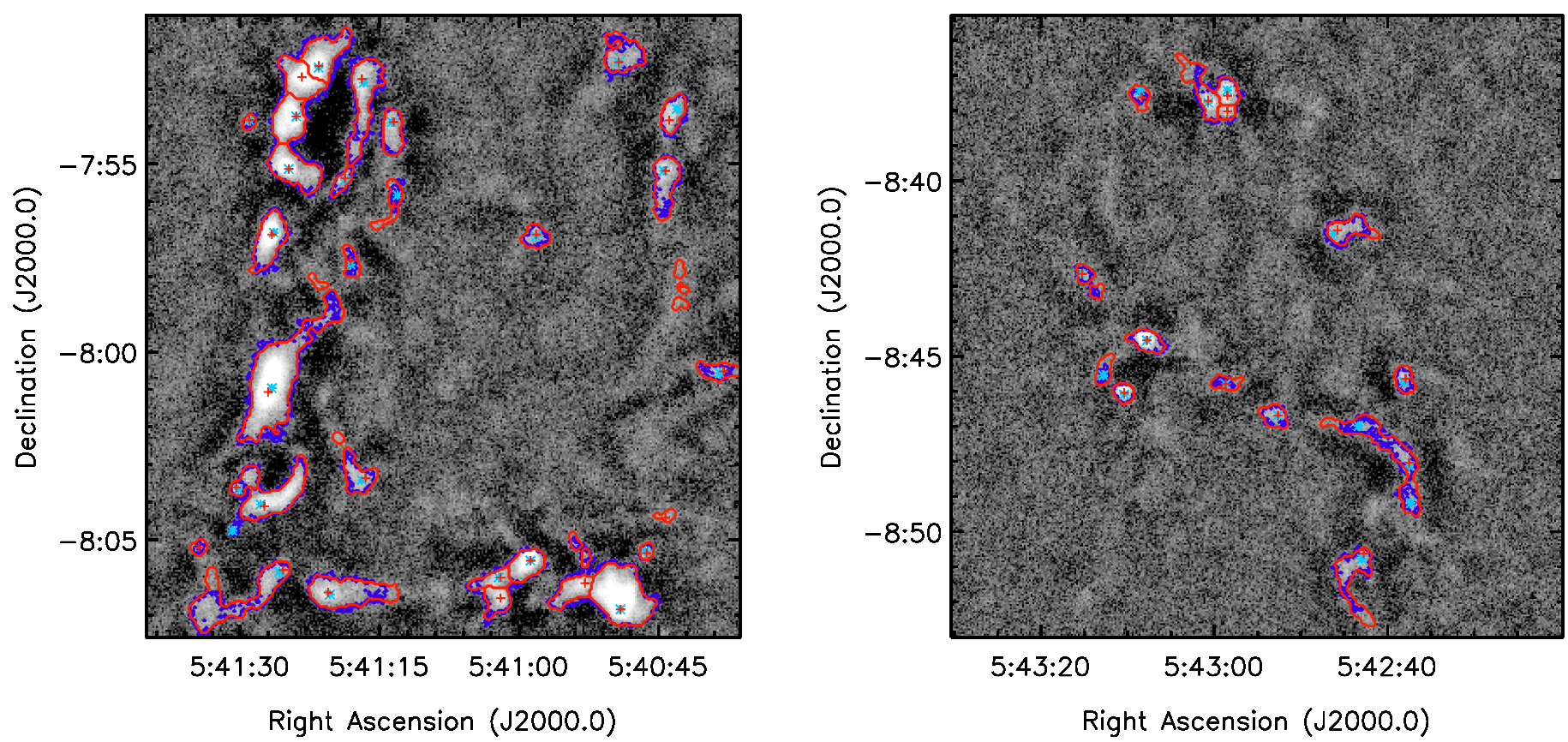

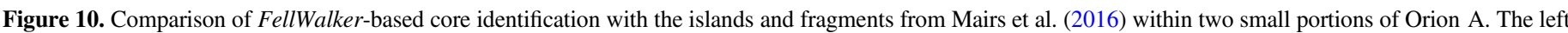

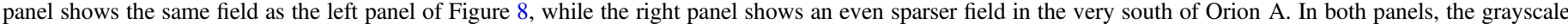

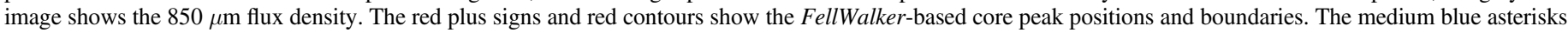

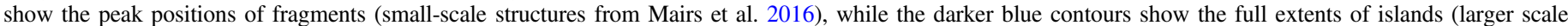
structures from Mairs et al. 2016).

and insignificant sources were also included in the initial catalog. The two main types of spurious sources that we aimed to remove were those present exclusively due to noise spikes near the edges of the map as well as small, faint sources that were most likely artifacts of background fluctuations. We eliminated these spurious sources by filtering the initial core list using an additional signal-to-noise requirement. We boxcar smoothed the original image using a 5 pixel wide window and removed sources whose peaks fell below two times the locally averaged rms noise per pixel. The smoothing and rms cutoff values were found to be the most effective combination after visually inspecting the results using a wide range of values. This process reduced the initial list of 1110 sources down to 773 robust core detections.

\section{A.1.3. Comparison of Getsources and Fellwalker Catalogs}

Unlike getsources, where a given map pixel may contribute flux density to multiple cores, FellWalker does not allow objects to overlap. As such, small and faint cores located beside large and bright cores have a greater chance of being missed, with their flux density being attributed to the larger core. This difference explains in part why we find fewer cores in the FellWalker catalog compared to the getsources extraction, despite similar post-extraction cuts being applied. Additionally, since getsources uses both 850 and $450 \mu \mathrm{m}$ data, the higher resolution of the latter map can help to de-blend cores that do not appear separate using the $850 \mu \mathrm{m}$ data alone.

A direct comparison of some of the dense cores identified using both getsources and FellWalker is shown in Figure 8. This figure highlights two different parts of Orion A: a less crowded field in the south, and a more crowded region near the ISF. It is apparent from Figure 8 that many of the cores have a reasonable correspondence, with similar peak positions. Although the sizes of the core contours are not directly comparable (since getsources-based cores would extend beyond their FWHM plotted), it is clear that getsources typically identifies the most peaked part of a structure, while FellWalker allows a much larger, often irregular, extent of fainter emission to be included. As discussed above, getsources also has a greater tendency to break up complex emission structures into more pieces. An example of this is the elongated structure at about (5:41:27, -8:00:00) in the left panel of Figure 8, where getsources identified two cores and FellWalker identified only one. For the faintest emission structures, there is a greater variation between the two catalogs. Factors such as the precise core boundaries have a larger impact on whether the inferred local signal-to-noise level is sufficient for the structure to be retained in the final catalog. An example of a core retained only in the FellWalker catalog can be seen in the left panel of Figure 8 at about (5:40:45, -8:04:00).

Quantitatively comparing the two catalogs, we find that 902 of 919 , or $98 \%$ of getsources-based cores, lie within a FellWalker-based core boundary, and 581 of 773 , or $75 \%$ of FellWalker-based cores, contain at least one getsources-based cores. These numbers reinforce the visual impression from the right-hand panel of Figure 8 that the majority of the variation between the two catalogs is caused by how emission is divided into cores, rather than whether emission is identified as belonging to any core.

\section{A.1.4. Comparison with Mairs et al.}

We furthermore compared our getsources-based core catalog with the structures identified in Mairs et al. (2016) who analyzed a similar SCUBA-2 map of Orion A; though, their analysis only extended as far north as the bottom of the ISF. Mairs et al. (2016) created two independent lists of structures within southern Orion A, large-scale islands and smaller-scale fragments, which typically lie within an island. The large-scale 


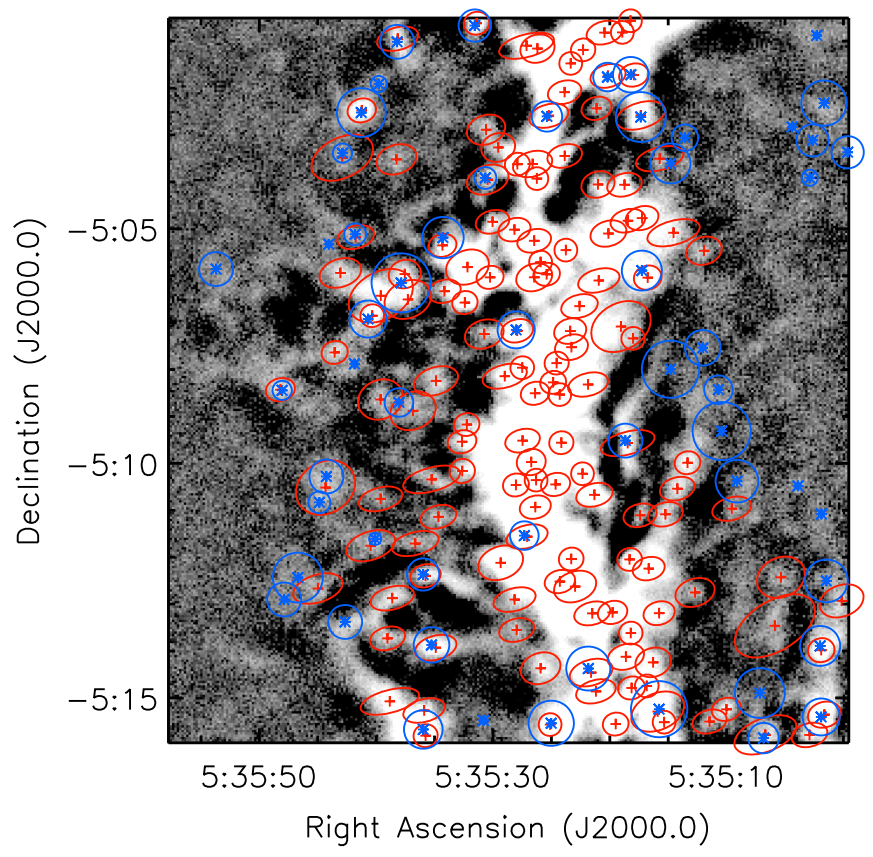

Figure 11. Comparison of our getsources-based core catalog with the starless and prestellar cores identified in Salji et al. (2015a). The area displayed is the same as the right panel of Figure 8 . The grayscale image shows the $850 \mu \mathrm{m}$ flux density. The red plus signs and larger ellipses show the getsources-based core peak positions and best-fit Gaussian FWHM contour. The blue asterisks and larger circles show the peak positions and sizes of the starless and prestellar cores from Salji et al. (2015a).

islands were identified using clumpfind (Williams et al. 1994), while the smaller-scale fragments were identified using a modified implementation of FellWalker. We compare our getsources-based cores across the same area in southern Orion A with both the island and fragment catalogs of Mairs et al. (2016) in Figure 9. The agreement between the catalogs is reasonably good. The left panel of Figure 9 appears to show a better correlation between the Mairs et al. (2016) structures and our getsources catalog than the similar comparison of getsources and FellWalker catalogs in Figure 9, but this trend is less obvious across the entire area mapped. For the larger scale islands, 485 of 518 , or $94 \%$ of the getsources-based cores in the southern portion of Orion A, lie within an island. Conversely, 244 of 364 , or $67 \%$ of the islands, contain one or more getsources-based cores. The $850 \mu \mathrm{m}$ map that Mairs et al. (2016) analyzed had better sensitivity to larger scale emission structures than the map we used, and we find that the Mairs et al. (2016) islands, which have no match in our getsourcesbased catalog, tend to be faint diffuse structures in our map with no peaks bright enough for getsources to pick up.

Moving to the smaller-scale fragment catalog of Mairs et al. (2016), we compared the peak positions of the fragments and getsources-based cores. Full coverage maps were not available for either the Mairs et al. (2016) fragments or for our getsources catalog (in the latter case since each pixel can contribute flux density to multiple cores). Therefore, we search for evidence of positional coincidence between the peak locations in each catalog. We find that 358 of 436 , or $82 \%$ of the fragments, lie within one getsources-based FWHM ${ }^{17}$, and very few additional cores lie within twice this value.

\footnotetext{
17 We used the geometric mean of the major and minor FWHM values returned by getsources.
}

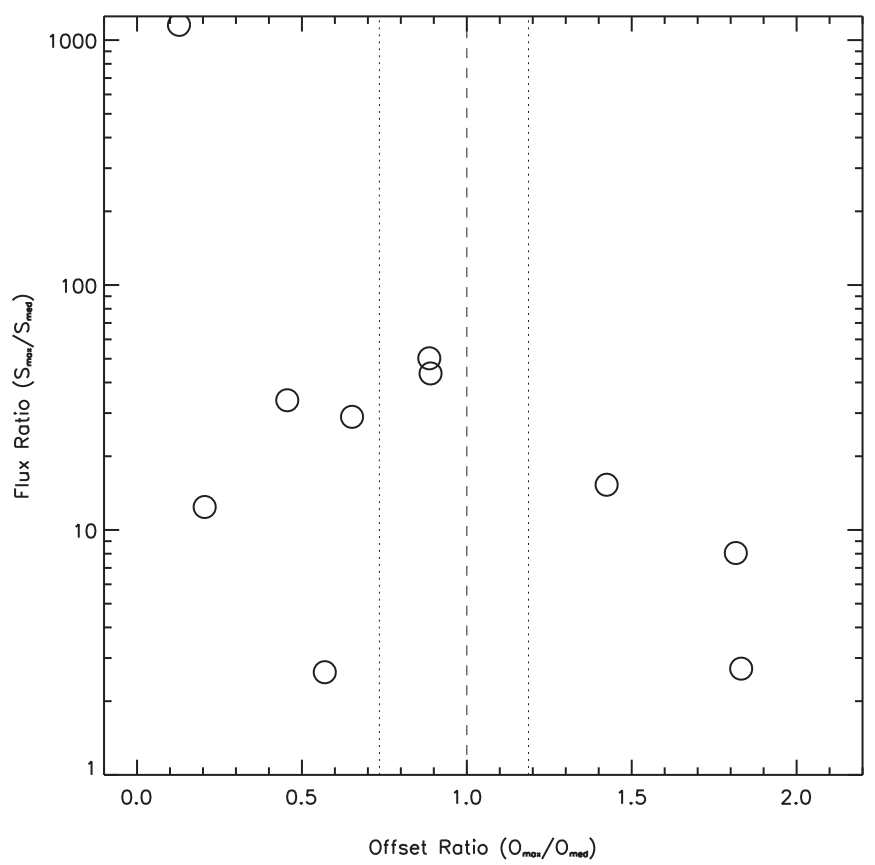

Figure 12. Comparison of the offset ratios derived for FellWalker-based clusters using a membership criterion of $N>10$ As with the original getsources-based analysis, most of the clusters have offset ratios less than one.

If we instead compare the Mairs et al. (2016) catalogs to our FellWalker-based catalog, as shown in Figure 10, we find a similar level of agreement. For the Mairs et al. (2016) islands, 408 of 514 , or $79 \%$ of our FellWalker-based cores, lie within an island, while 290 of 364, or $80 \%$, of the Mairs et al. (2016) islands, contain at least one FellWalker-based core. Meanwhile, all of the Mairs et al. (2016) fragments lie within our FellWalker-based core boundaries. Mairs et al. (2016) applied FellWalker in a different configuration than our work to identify their fragments, so it is at first surprising that the agreement with our FellWalker catalog is not even better. A careful visual inspection of the two catalogs and maps used for identification shows that most of the fragments (and islands) with no correspondence in our FellWalker (or getsources) catalogs are indeed faint extended structures for which the map we analyze has less sensitivity. For the Mairs et al. (2016) sources that we do detect with FellWalker, the agreement is very good.

\section{A.1.5. Comparison with Salji et al.}

Finally, we compare our getsources-based core catalog with the 432 starless and prestellar cores identified in the area around the ISF by Salji et al. (2015a) using a new core identification algorithm first presented in that work. Figure 11 shows a comparison of the two catalogs in the same field as is shown in the right panel of Figure 8. The correspondence of cores here is much poorer, with only 161 of the 432 Salji et al. cores lying within the FWHM extent of a getsources-based core. There are a variety of factors contributing to this poor match. The first is related to the constraints Salji et al. placed on the cores they identified. Namely, their algorithm was tuned to explicitly detect only nearly circular cores with sizes ranging between about 0.03 and $0.1 \mathrm{pc}$ (elongated structures were instead analyzed as filaments in Salji et al. 2015b). 

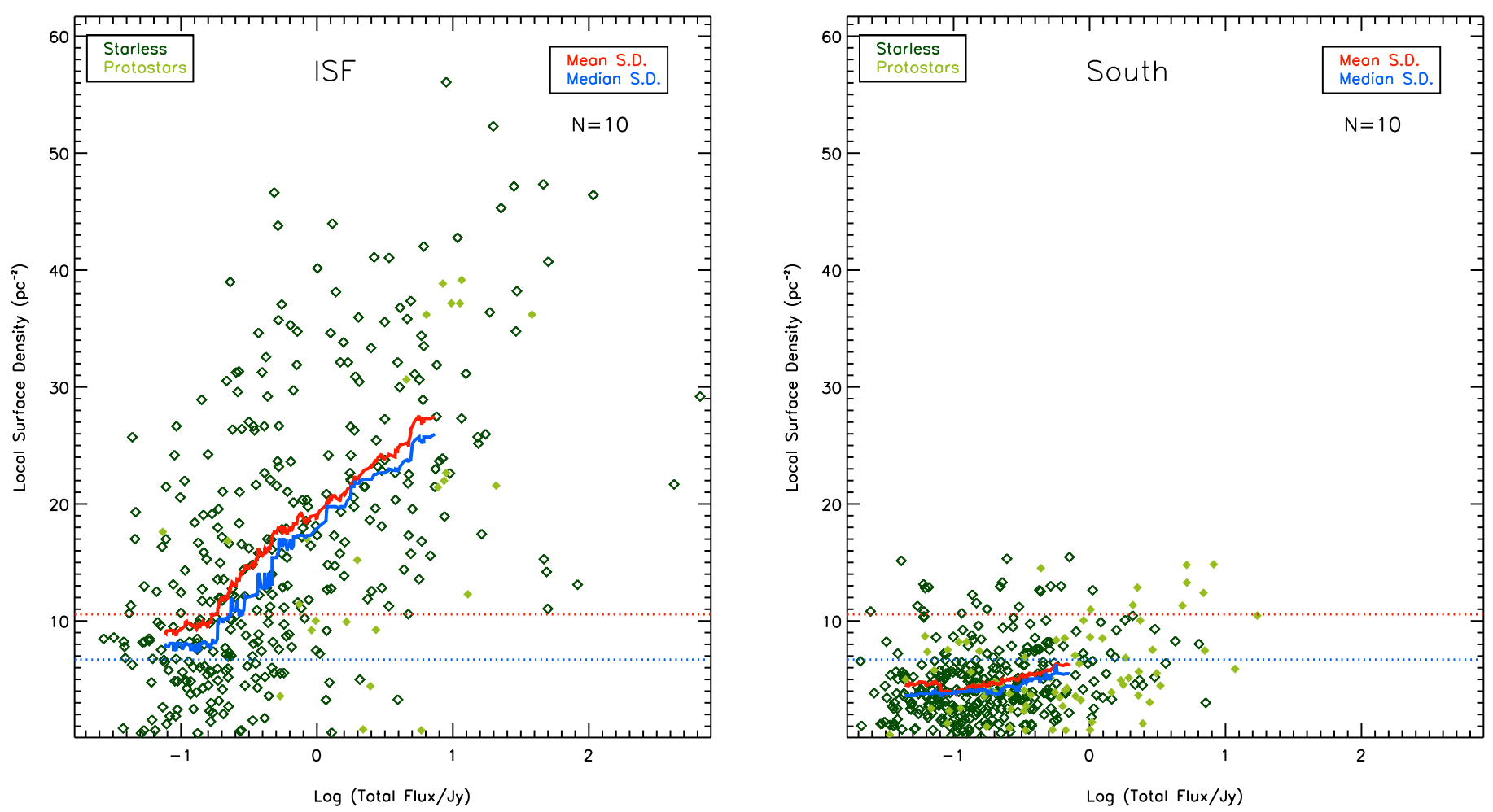

Figure 13. Local core-core surface density vs. total core flux density for the dense cores identified using FellWalker. Similar to the getsources-based analysis shown in Figure 7, there is a strong positive trend between total flux density and core-core surface density for both the ISF and southern Orion A. See Figure 7 for the plotting conventions used.

Furthermore, the 48 cores associated with a protostar were removed from Salji et al.'s analysis and their properties (including location) were not reported. It seems likely that these factors (size and shape constraints and protostar elimination) drive the poor correspondence between cores along the central ISF spine. Furthermore, as noted in Section 2, Salji et al. (2015a) analyzed a limited portion of the full data set across the northern portion of Orion A, using an earlier data reduction process that was less sensitive to large-scale structure. The combination of these two effects drives a poorer correspondence between cores identified in the outskirts of the central ISF spine where faint sources are more prevalent.

\section{A.2. MST-Based Cluster Analysis}

Recognizing that the differences in source identification could impact the results of our cluster analysis, we re-ran all of our analyses using the FellWalker-based catalog. Here, we discuss the impact on the MST-based analysis, namely, the distribution of offset ratios. We follow the same procedure as outlined in Section 4 to use an MST to identify clusters, and find a best-fit value of $L_{\text {crit }}$ of $0.33 \mathrm{pc}$ (with a full range of acceptable fits of $0.31-0.37 \mathrm{pc}$ ). FellWalker tends to split up regions of complex emission structure like the ISF into fewer cores than getsources, which resulted in ISF cores having relatively less influence on the distribution of branch lengths in the region. Given this difference, a single global fit to the entire Orion A distribution of branch lengths provided visually reasonable MST-based clusters across the entire field, unlike in our getsources-based analysis.
Using the standard membership criterion of $N>10$, we identify 10 clusters across Orion A. Most of these clusters (8 of 10) have a very good correspondence to the getsources-based clusters. In general, the FellWalker-based clusters have fewer members, due to clustered emission being divided into fewer cores in FellWalker. Similar to the getsources-based clusters, most of the FellWalker-based clusters have offset ratios less than one, with only 3 of 10 having offset ratios greater than one, as illustrated in Figure 12.

\section{A.3. $S-\Sigma$ Analysis}

We next examine the relationship between total flux density and core-core surface density for the FellWalker-based cores and compare with the results seen in Section 5 for the getsources-based core catalog. Figure 7 shows the $S-\Sigma$ plot for the FellWalker-based catalog, using the 10 nearest neighbors to estimate the core-core surface density (NN10) as was done for the getsources-based analysis shown in Figure 7. Clearly, the same trend for higher total flux densities with increased surface density of neighbors is seen. As with the getsources-based analysis, the two-sample KS test gives an extremely low probability that the higher and lower flux density starless or protostellar cores have a similar distribution of surface densities in either the ISF or southern Orion A, for NN5 or NN10 and either of the high versus low splits tested in Section 5. Similarly, the Mann-Whitney test shows a strong probability of higher flux density cores inhabiting higher corecore surface density environments. Therefore, with this metric too, the choice of core identification method has no effect on our conclusions. 

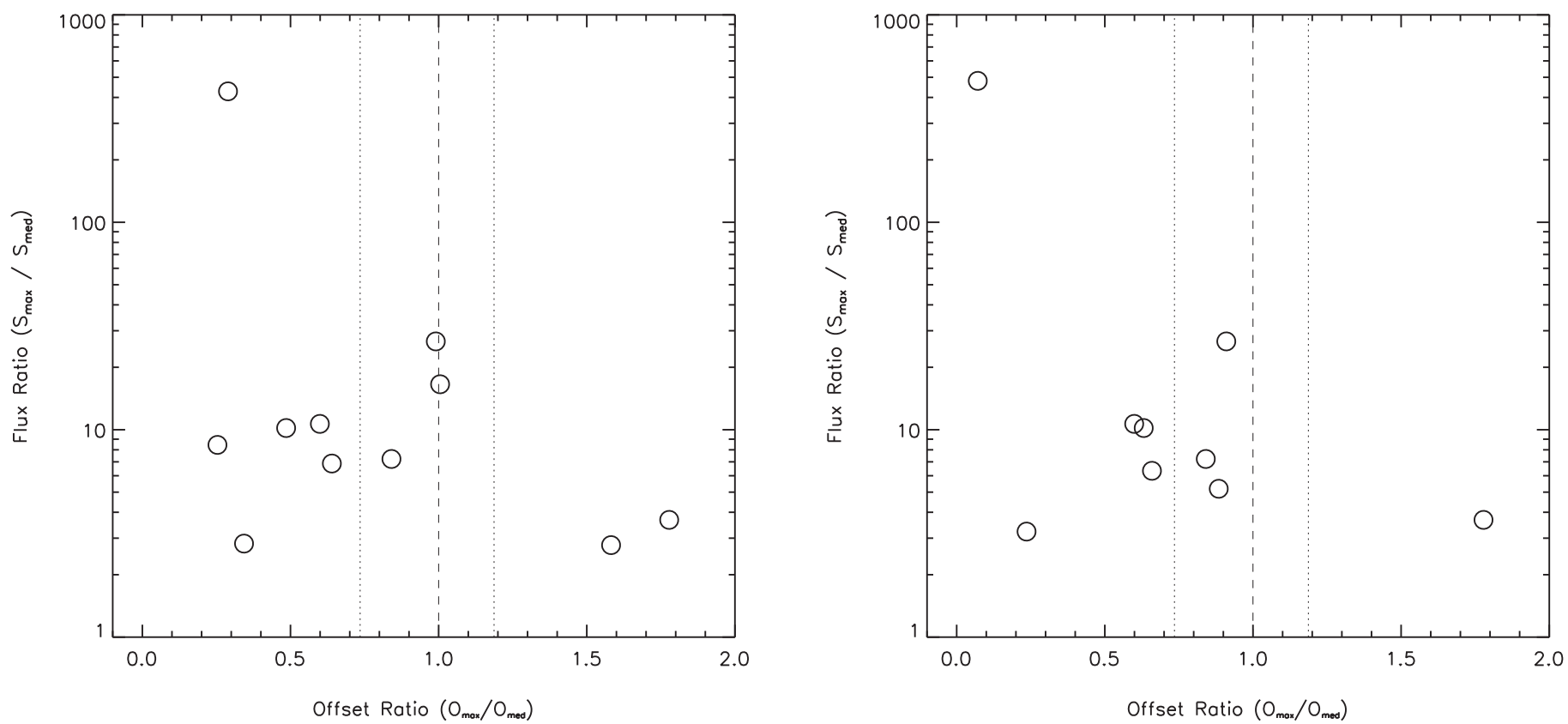

Figure 14. Comparison of offset ratios measured for getsources-based clusters using the minimum (left) and maximum (right) values of $L_{\text {crit }}, 0.32$ and $0.40 \mathrm{pc}$, respectively. As with the original analysis, the majority of clusters have offset ratios smaller than one.

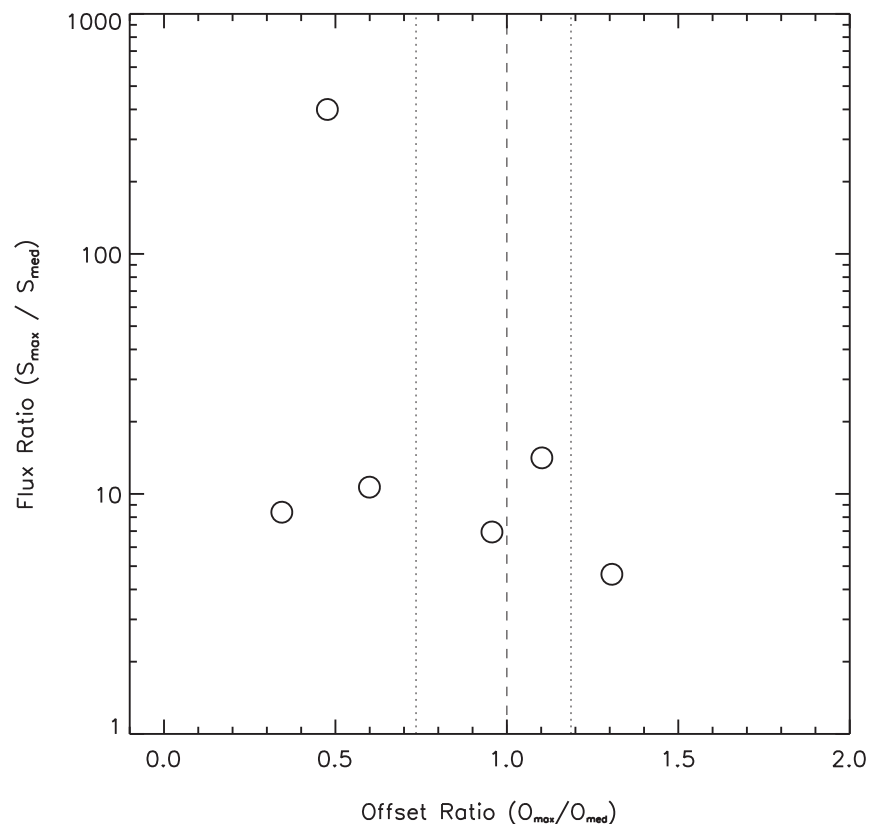

Figure 15. Comparison of offset ratios measured for getsources-based clusters using the $L_{\text {crit }}$ value derived for the entire Orion A core population of $0.22 \mathrm{pc}$. Again, the majority of clusters have offset ratios less than one.

\section{APPENDIX B \\ UNCERTAINTIES IN GETSOURCES-BASED MST CLUSTERS}

As also discussed in K16, use of the MST technique requires several user-defined parameters, which have the potential to influence the resulting clusters, and hence, the analysis. We examine the influence of varying $L_{\text {crit }}$ and $N$, the minimum number of cluster members, in turn. We find that, although the details of the clusters identified vary with changes to these parameters, the resulting offset ratios show little variation, and hence our conclusions are robust.

\section{B.1. Variations in $L_{\text {crit }}$}

Here, we examine the effect of the uncertainty in $L_{\text {crit }}$ on the resulting clusters and analysis. Our range of uncertainty in $L_{\text {crit }}$ based on the cumulative branch length distribution measured in the southern part of Orion A is $0.32-0.40 \mathrm{pc}$. We re-ran our analysis using both of these values. Qualitatively, the clusters appear similar over the range of $L_{\text {crit }}$ values tested. Adopting $L_{\text {crit }}=0.32 \mathrm{pc}$, only clusters 1,3 , and 5 show any changes in their membership, and these are restricted to small percentages of members located at the cluster peripheries. The changes are slightly larger for $L_{\text {crit }}=0.40 \mathrm{pc}$, where this increase merges several formerly distinct clusters $(2,6$, and 9$)$ into the large ISF cluster 1 , and a new cluster that barely meets the relaxed criteria is identified. The remaining clusters $(3,4,5,7,8$, and 10), however, still have identical membership. We ran our offset ratio analysis on the clusters identified using the minimum and maximum $L_{\text {crit }}$ found in the south, and show these results in Figure 14. As can be clearly seen in the figure, most clusters have offset ratios less than one under both scenarios.

We also consider a more extreme variation in $L_{\text {crit }}$. As discussed in Section 4.1, our initial MST analysis of the entire Orion A dense core population indicated a smaller $L_{\text {crit }}$ value of $0.22 \mathrm{pc}$, which was strongly dominated by cores around the ISF. Using this $L_{\text {crit }}$ value instead noticeably shrinks the membership of clusters $1,2,6,7$, and 9, and reduces clusters 4 , $5,8,10$, and 11 to below the minimum number of cluster members required, while only cluster 3 remains identical. Even with these extreme changes to the cluster membership, we find the resulting offset ratios tend to be less than one, as shown in Figure 15.

It is encouraging to note that a large range of $L_{\text {crit }}$ values, including the full-cluster value that excludes most of the clusters in the southern part of Orion A from the analysis, provide similar and consistent results. We conclude that our overall results are therefore robust to reasonable permutations in cluster definition. 

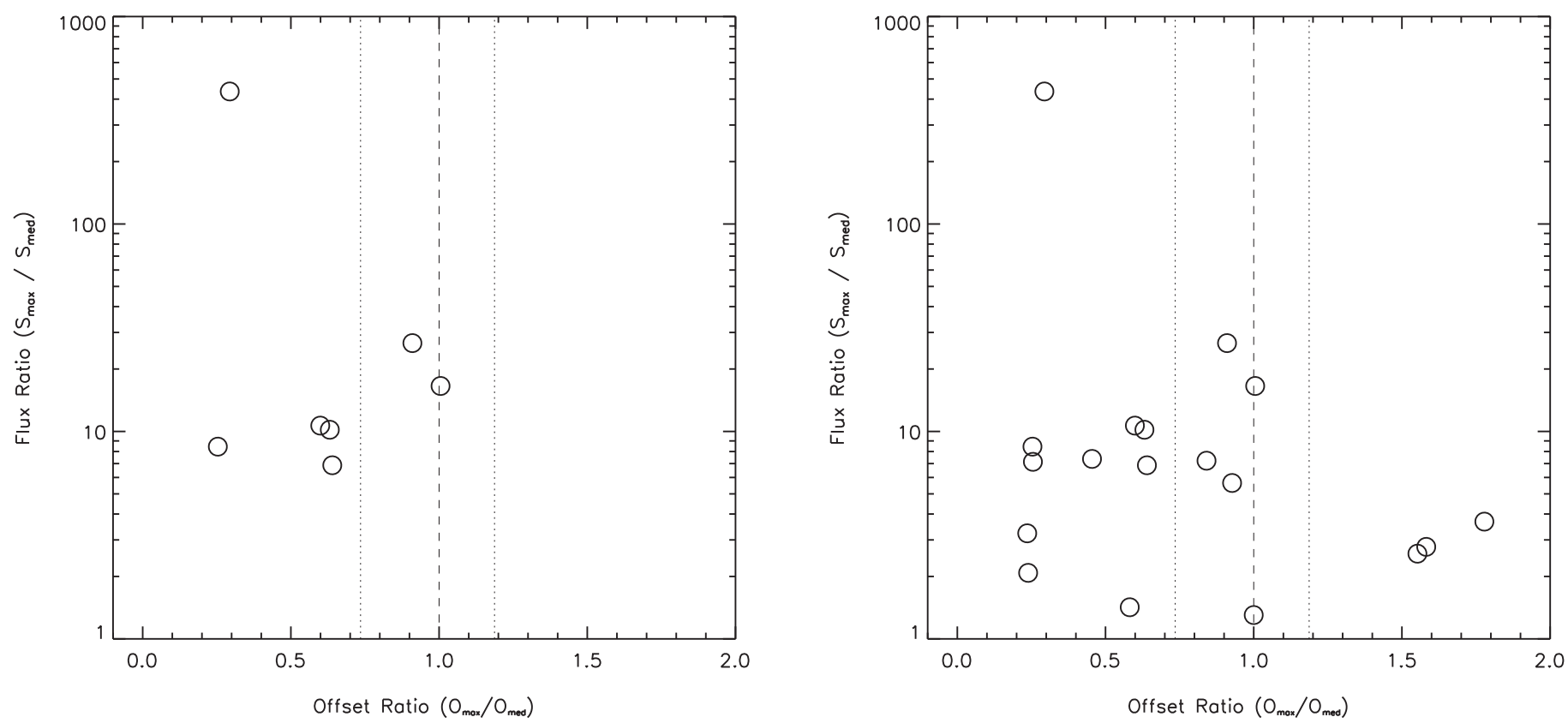

Figure 16. Comparison of offset ratios for getsources-based clusters using a membership criterion of $N>15$ (left) and $N>5$ (right). As with the original analysis, the majority of clusters have offset ratios less than one.
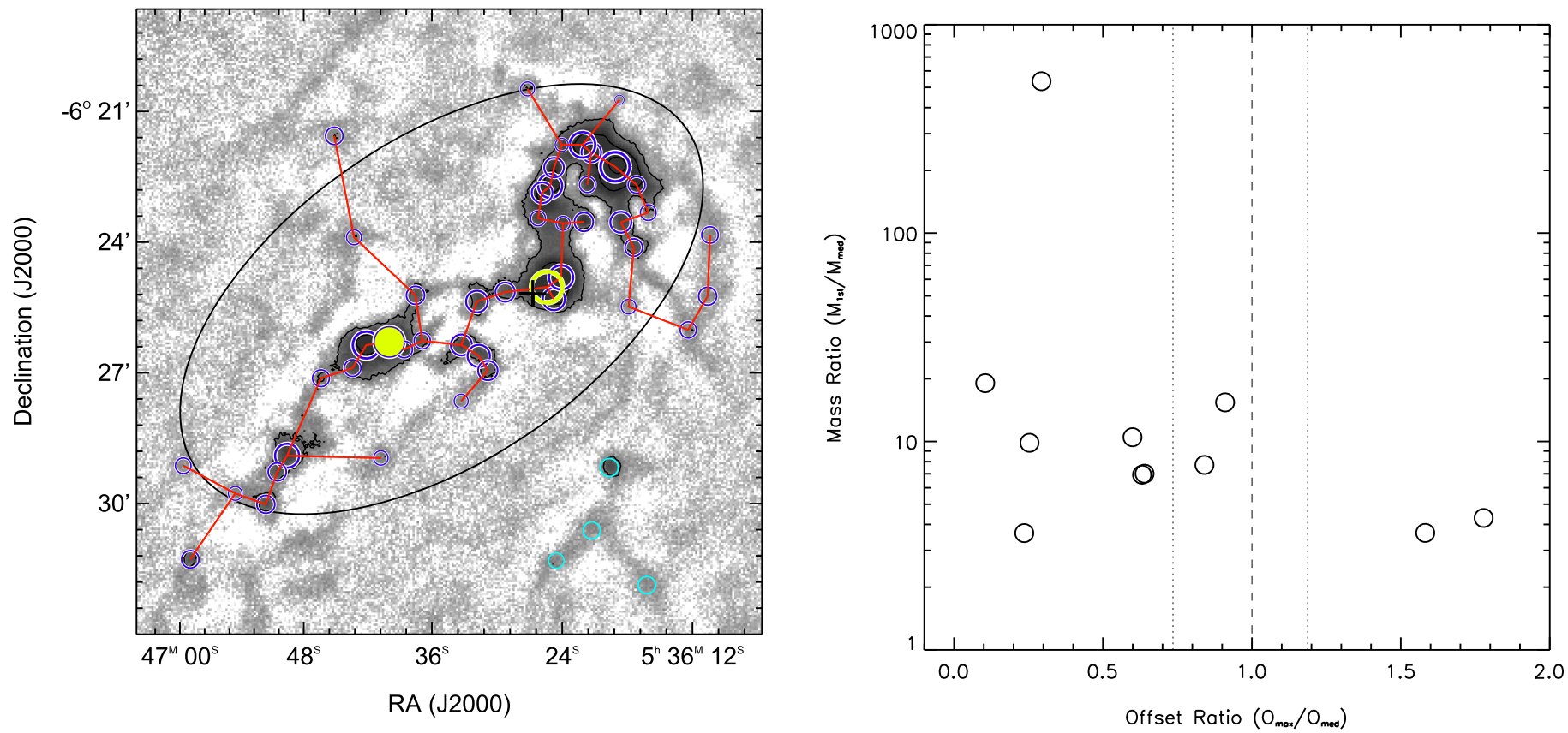

Figure 17. Left: the minimal spanning tree structure for cluster 2, using temperatures from Lombardi et al. (2014) to estimate the mass rankings of the dense cores. The most massive (protostellar) core is denoted with the thick yellow open circle, and is notably different from the position of the highest flux (protostellar) core in the cluster 2 shown in Figure 4. Right: the distribution of offset ratios measured for the getsources-based clusters, using the most massive dense core in each cluster, showing nearly identical offset ratios to those shown in the left panel of Figure 6 based on the highest flux cluster member.

\section{B.2. Variations in $N$}

Next, we consider the effect of adopting a different requirement for the minimum number of cluster members. Our main analysis requires $N>10$, but here we present results for $N>15$ and $N>5$. The $N>15$ clusters are a subset of the original $N>10$ clusters, with 4 of the 11 initially identified clusters excluded with this larger membership criterion. The $N>15$ set of clusters show an even stronger tendency than our full $N>10$ sample for offset ratios below one, with six of the seven having ratios much less than one, as can be seen in the left panel of Figure 16. Extending the MST-based clusters to those with $N>5$ members adds a further seven "clusters" to the original $N>10$ sample. We note that some of these additional clusters are extremely small, with three containing only six members. At such a small- $N$ limit, measuring the offset ratio becomes more uncertain. The cluster center, for example, is much more prone to variations with the inclusion or exclusion of cluster members. Despite this increased uncertainty, most of the additional clusters added when extending the sample to $N>5$ do still have offset ratios below one, as shown 

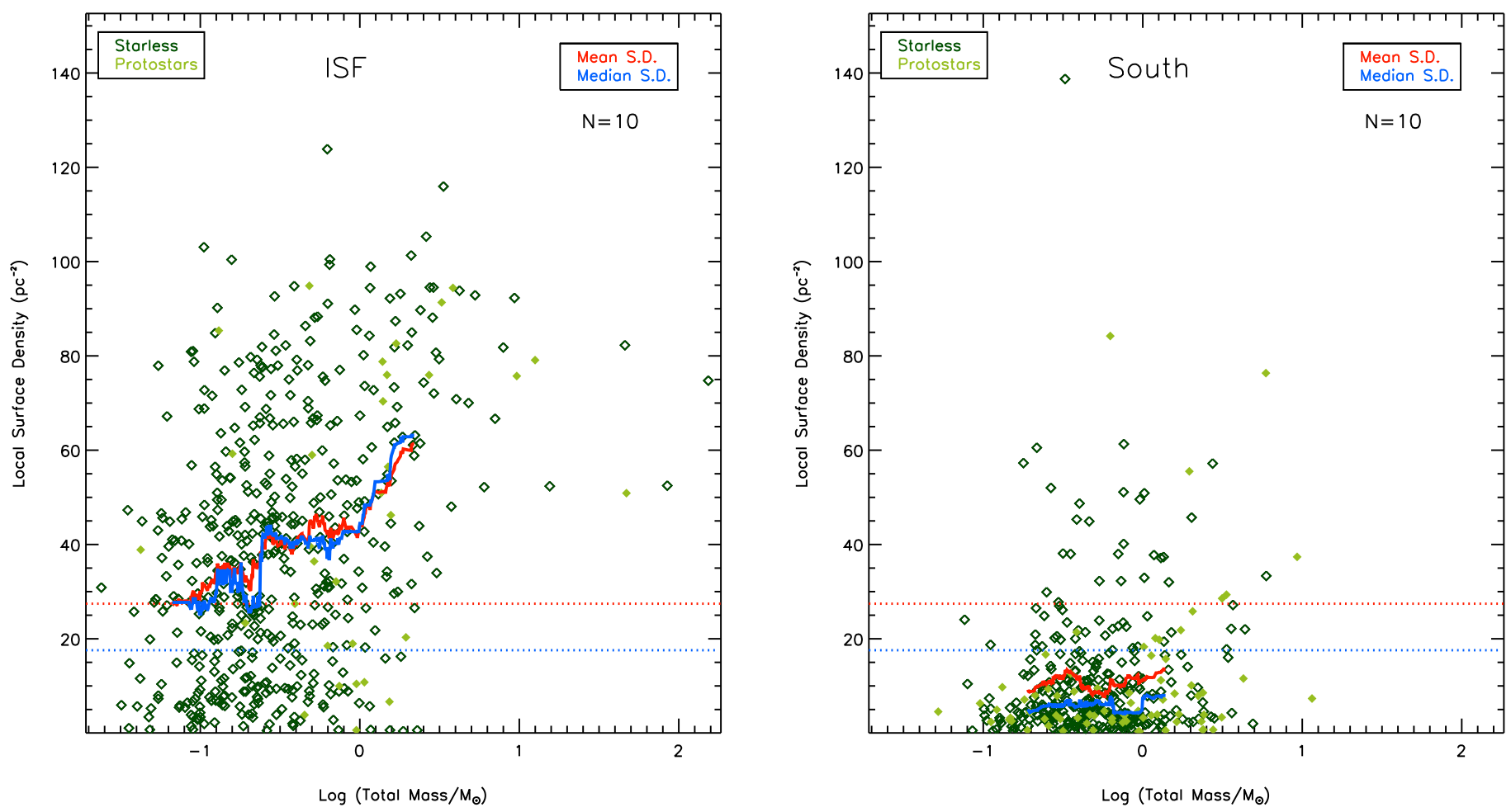

Figure 18. Core-core surface density as a function of their estimated total mass, using temperatures from Lombardi et al. (2014). Similar to Figure 7, higher mass cores tend to be found in more clustered environments, with this trend being especially strong in the ISF.

in the right panel of Figure 16. We therefore conclude that the offset ratios measured for the getsources-based clusters are robust to variations within the user-defined parameters of the MST method.

\section{APPENDIX C EFFECT OF USING HERSCHEL TEMPERATURES FOR MASS ESTIMATES}

We also ran our analysis on the dense core masses, as estimated using Equation (1) with dust temperatures measured by Lombardi et al. (2014) using Herschel data. For simplicity, we adopt a constant temperature for each dense core, using the value that Lombardi et al. (2014) measure at each dense core's peak. The mean and median temperature measured for the getsources-based dense cores are $22.6 \mathrm{~K}$ and $19.3 \mathrm{~K}$, respectively, while the full range is $11.7-65.5 \mathrm{~K}$. It is important to note, however, that the Lombardi et al. (2014) temperature analysis does not extend to wavelengths longer than those covered by Herschel. Sadavoy et al. (2013) analyzed Herschel and SCUBA-2 observations in the Perseus molecular cloud, and found that the inclusion of the longer-wavelength SCUBA2 data improved temperature estimates by $40 \%$ over the best that could be obtained using Herschel data alone. The improved angular resolution at longer wavelengths and better sensitivity of SCUBA-2 to cold dust are two of the driving factors in the improvement to the temperature estimate that Sadavoy et al. (2013) found. Many star-forming regions may have dust at multiple temperatures along the same line of sight, and observations at the shortest Herschel wavelengths will have greater sensitivity to any diffuse population of hot dust that is present. Since the Lombardi et al. (2014) temperature estimates did not include SCUBA-2 data, the temperature estimates for some of our SCUBA-2 dense cores may be too high, biased by the higher temperatures of surrounding lower density material. Nonetheless, the Herschel-based temperature provide us an important avenue beyond the starless/protostellar core separation employed in the body of the text, to test the validity of the relative core mass rankings assumed.

\section{C.1. Offset Ratios for MST-based Clusters}

We re-examined the offset ratio analysis of Section 4 using instead the mass-ranking of the cores to identify the most massive cluster member. In all but one cluster (cluster 2), the most massive cluster member is identical to the highest flux cluster member, and therefore the offset ratios we measure remain identical. In cluster 2 , the most massive cluster member lies closer to the cluster center than the highest flux cluster member did, creating a slightly larger population of small offset ratio clusters than presented in the main paper. Figure 17 shows the distribution of offset ratios obtained when we use the massranking of dense cores. With the revised offset ratio distribution, we find a less than $2 \%$ probability that the most massive cluster members are randomly located within their clusters.

\section{C.2. M-sigma}

We also ran the $S-\Sigma$ analysis of Section 5 based instead on the estimated masses for all of the dense cores. Figure 18 shows the local core-core surface density as a function of dense core mass for the cores in the ISF and the south separately. As we saw in Figure 7, both regions show a significant trend between increasing mass (/flux) and increasing core-core surface density. Statistically, we find more significant results for all of the tests reported here than when using the dense core fluxes reported in Section 5, due to the increased sample size (since here we can examine starless and protostellar cores 
simultaneously). In other words, we find that the core-core surface densities are significantly different for the higher and lower mass dense cores, with a strong tendency for higher core-core surface densities being associated with higher mass dense cores.

We therefore conclude that our clustering analysis is unaffected by using masses derived from Herschel-based temperatures for the cores in this study.

\section{REFERENCES}

Allison, R. J., Goodwin, S. P., Parker, R. J., et al. 2009a, ApJL, 700, L99 Allison, R. J., Goodwin, S. P., Parker, R. J., et al. 2009b, MNRAS, 395, 1449 Allison, R. J., Goodwin, S. P., Parker, R. J., Portegies Zwart, S. F., \& de Grijs, R. 2010, MNRAS, 407, 1098

André, P., Di Francesco, J., Ward-Thompson, D., et al. 2014, in Protostars and Planets VI, ed. H. Beuther et al. (Tuscon, AZ: Univ. of Arizona Press), 27

Bally, J. 2008, in Handbook of Star Forming Regions: Vol. I, The Northern Sky, ed. B. Reipurth et al. (San Francisco, CA: ASP), 459

Bally, J., Langer, W. D., Stark, A. A., \& Wilson, R. W. 1987, ApJL, 312, L45

Bergin, E. A., \& Tafalla, M. 2007, ARA\&A, 45, 339

Berry, D. S. 2013, StaUN, 255

Berry, D. S. 2015, A\&C, 10, 22

Berry, D. S., Reinhold, K., Jenness, T., \& Economou, F. 2007, in ASP Conf. Ser. 376, Astronomical Data Analysis Software and Systems XVI, ed. R. A. Shaw, F. Hill, \& D. J. Bell (San Francisco, CA: ASP), 425

Bintley, D., Holland, W. S., MacIntosh, M. J., et al. 2014, Proc. SPIE, 9153, 915303

Bonnell, I. A., Bate, M. R., Clarke, C. J., \& Pringle, J. E. 2001, MNRAS, 323,785

Bonnell, I. A., \& Davies, M. B. 1998, MNRAS, 295, 691

Bressert, E., Bastian, N., Gutermuth, R., et al. 2010, MNRAS, 409, L54

Buckle, J. V., Davis, C. J., Francesco, J. D., et al. 2012, MNRAS, 422, 521

Carpenter, J. M., Meyer, M. R., Dougados, C., Strom, S. E., \& Hillenbrand, L. A. 1997, AJ, 114, 198

Cartwright, A., \& Whitworth, A. P. 2004, MNRAS, 348, 589

Casertano, S., \& Hut, P. 1985, ApJ, 298, 80

Chapin, E. L., Berry, D. S., Gibb, A. G., et al. 2013, MNRAS, 430, 2545

Currie, M. J., Berry, D. S., Jenness, T., et al. 2014, in ASP Conf. Ser. 485, Astronomical Data Analysis Software and Systems XXIII, ed. N. Manset \&

P. Forshay (San Francisco, CA: ASP), 391

Davidge, T. J. 2015, PASP, 127, 836

Dempsey, J. T., Friberg, P., Jenness, T., et al. 2013, MNRAS, 430, 2534

Elmegreen, B. G., Hurst, R., \& Koenig, X. 2014, ApJL, 782, L1

Enoch, M. L., Evans, N. J., II, Sargent, A. I., et al. 2008, ApJ, 684, 1240

Forbrich, J., Rivilla, V. M., Menten, K. M., et al. 2016, arXiv:1603.05666

Gennaro, M., Brandner, W., Stolte, A., \& Henning, T. 2011, MNRAS, 412, 2469

Girichidis, P., Federrath, C., Allison, R., Banerjee, R., \& Klessen, R. S. 2012, MNRAS, 420, 3264

Gutermuth, R. A., Megeath, S. T., Myers, P. C., et al. 2009, ApJS, 184, 18

Hatchell, J., Fuller, G. A., Richer, J. S., Harries, T. J., \& Ladd, E. F. 2007, A\&A, 468, 1009

Hillenbrand, L. A. 1997, AJ, 113, 1733

Holland, W. S., Bintley, D., Chapin, E. L., et al. 2013, MNRAS, 430, 2513

Hsu, W.-H., Hartmann, L., Allen, L., et al. 2012, ApJ, 752, 59

Hsu, W.-H., Hartmann, L., Allen, L., et al. 2013, ApJ, 764, 114

Hunter, T. R., Brogan, C. L., Cyganowski, C. J., \& Young, K. H. 2014, ApJ, 788,187
Hunter, T. R., Brogan, C. L., Megeath, S. T., et al. 2006, ApJ, 649, 888 Johnstone, D., \& Bally, J. 1999, ApJL, 510, L49

Johnstone, D., \& Bally, J. 2006, ApJ, 653, 383

Kainulainen, J., Stutz, A. M., Stanke, T., et al. 2016, arXiv:1603.05688

Kim, M. K., Hirota, T., Honma, M., et al. 2008, PASJ, 60, 991

Kirk, H., Di Francesco, J., Johnstone, D., et al. 2016a, ApJ, 817, 167

Kirk, H., Johnstone, D., Di Francesco, J., et al. 2016b, ApJ, 821, 98

Kirk, H., Johnstone, D., \& Tafalla, M. 2007, ApJ, 668, 1042

Kirk, H., \& Myers, P. C. 2011, ApJ, 727, 64

Kirk, H., Offner, S. S. R., \& Redmond, K. J. 2014, MNRAS, 439, 1765

Kirk, H., Pineda, J. E., Johnstone, D., \& Goodman, A. 2010, ApJ, 723, 457

Kirk, J. M., Ward-Thompson, D., \& André, P. 2005, MNRAS, 360, 1506

Könyves, V., André, P., Men'shchikov, A., et al. 2015, A\&A, 584, A91

Kounkel, M., Megeath, S. T., Poteet, C. A., Fischer, W. J., \& Hartmann, L. 2016, ApJ, 821, 52

Kryukova, E., Megeath, S. T., Gutermuth, R. A., et al. 2012, AJ, 144, 31

Lada, C. J., \& Lada, E. A. 2003, ARA\&A, 41, 57

Landsman, W. B. 1993, in ASP Conf. Ser. 52, Astronomical Data Analysis Software and Systems II, ed. R. J. Hanisch, R. J. V. Brissenden, \& J. Barnes (San Francisco, CA: ASP), 246

Lombardi, M., Bouy, H., Alves, J., \& Lada, C. J. 2014, A\&A, 566, A45

Mairs, S., Johnstone, D., Kirk, H., et al. 2015, MNRAS, 454, 2557

Mairs, S., Johnstone, D., Kirk, H., et al. 2016, MNRAS, 461, 4022

Maschberger, T., \& Clarke, C. J. 2011, MNRAS, 416, 541

McKee, C. F., \& Tan, J. C. 2003, ApJ, 585, 850

Megeath, S. T., Gutermuth, R., Muzerolle, J., et al. 2012, AJ, 144, 192

Megeath, S. T., Wilson, T. L., \& Corbin, M. R. 2005, ApJL, 622, L141

Meingast, S., Alves, J., Mardones, D., et al. 2016, A\&A, 587, A153

Men'shchikov, A., André, P., Didelon, P., et al. 2012, A\&A, 542, A81

Menten, K. M., Reid, M. J., Forbrich, J., \& Brunthaler, A. 2007, A\&A, 474,515

Muench, A., Getman, K., Hillenbrand, L., \& Preibisch, T. 2008, in Star Formation in the Orion Nebula I: Stellar Content, ed. B. Reipurth (San Francisco, CA: ASP), 483

Myers, P. C. 2009, ApJ, 700, 1609

Myers, P. C. 2011, ApJ, 743, 98

Parker, R. J., \& Goodwin, S. P. 2015, MNRAS, 449, 3381

Parker, R. J., Maschberger, T., \& Alves de Oliveira, C. 2012, MNRAS, 426, 3079

Polychroni, D., Schisano, E., Elia, D., et al. 2013, ApJL, 777, L33

Sadavoy, S. I., Di Francesco, J., Johnstone, D., et al. 2013, ApJ, 767, 126

Salji, C. J., Richer, J. S., Buckle, J. V., et al. 2015a, MNRAS, 449, 1769

Salji, C. J., Richer, J. S., Buckle, J. V., et al. 2015b, MNRAS, 449, 1782

Schneider, N., Csengeri, T., Hennemann, M., et al. 2012, A\&A, 540, L11

Shu, F. H., Adams, F. C., \& Lizano, S. 1987, ARA\&A, 25, 23

Smith, R. J., Longmore, S., \& Bonnell, I. 2009, MNRAS, 400, 1775

Stolte, A., Brandner, W., Brandl, B., \& Zinnecker, H. 2006, AJ, 132, 253

Stutz, A. M., \& Gould, A. 2016, A\&A, 590, A2

Stutz, A. M., Tobin, J. J., Stanke, T., et al. 2013, ApJ, 767, 36

Takahashi, S., Ho, P. T. P., Teixeira, P. S., Zapata, L. A., \& Su, Y.-N. 2013, ApJ, 763, 57

Tan, J. C., Beltrán, M. T., Caselli, P., et al. 2014, in Protostars and Planets VI, ed. H. Beuther et al. (Tuscon, AZ: Univ. Arizona Press), 149

Walsh, A. J., Myers, P. C., \& Burton, M. G. 2004, ApJ, 614, 194

Walsh, A. J., Myers, P. C., Di Francesco, J., et al. 2007, ApJ, 655, 958

Ward-Thompson, D., Di Francesco, J., Hatchell, J., et al. 2007, PASP, 119, 855

Ward-Thompson, D., Pattle, K., Kirk, J. M., et al. 2016, MNRAS, 463, 1008

Williams, J. P., de Geus, E. J., \& Blitz, L. 1994, ApJ, 428, 693

Wright, N. J., Parker, R. J., Goodwin, S. P., \& Drake, J. J. 2014, MNRAS, 438,639

Zinnecker, H., \& Yorke, H. W. 2007, ARA\&A, 45, 481 